\title{
Drug-free mannosylated liposomes inhibit tumor growth by promoting the polarization of tumor-associated macrophages
}

This article was published in the following Dove Press journal: International Journal of Nanomedicine

\author{
Jun $\mathrm{Ye}^{\mathrm{I-3}}$ \\ Yanfang Yang ${ }^{1,2}$ \\ Wujun Dong ${ }^{1,2}$ \\ Yue Gao ${ }^{1,2}$ \\ Yingying Meng ${ }^{1,2}$ \\ Hongliang Wang ${ }^{1,2}$ \\ Lin $L i^{1,2}$ \\ Jing Jin ' \\ Ming $\mathrm{Ji}^{1}$ \\ Xuejun $\mathrm{Xia}^{1,2}$ \\ Xiaoguang Chen' \\ Yiqun Jin ${ }^{3}$ \\ Yuling Liu ${ }^{1,2}$

\begin{abstract}
'State Key Laboratory of Bioactive Substance and Function of Natural Medicines, Institute of Materia Medica, Chinese Academy of Medical Sciences \& Peking Union Medical College, Beijing 100050, People's Republic of China; Technology and Novel Formulation, Institute of Materia Medica, Chinese Academy of Medical Sciences \& Peking Union Medical College, Beijing 100050, People's Republic of China; ${ }^{3}$ Research and Development Department, Beijing Wehand-bio Pharmaceutical Co. Ltd, Beijing, 102600, People's Republic of China
\end{abstract} \\ ${ }^{2}$ Beijing Key Laboratory of Drug Delivery
}

Correspondence: Yuling Liu State Key Laboratory of Bioactive Substance and Function of Natural Medicines, Institute of Materia Medica, Chinese Academy of Medical Sciences \& Peking Union Medical College, I

Xiannongtan Street, Beijing I00050,

People's Republic of China

Tel +86 1089285188

Fax +86 1089285190

Email ylliu@imm.ac.cn
Background: Tumor-associated macrophages (TAMs) are critical in tumor progression and metastasis. Selective targeting of TAMs holds great potential to ameliorate the immunosuppressive tumor microenvironment and enhance the efficacy of antitumor therapy. Various liposomes have been developed to target TAMs via cell-specific surface receptors either to deplete or re-educate TAMs. Since immuno-stimulation often initiates with the interaction of nanocarriers with the innate immunity cells such as macrophages, the intrinsic impact of drug-free liposomes on macrophage activation and polarization via cell interaction is one of the most critical issues in nanomedicine for promoting effective immunotherapy.

Methods: In this study, conventional bare liposomes, PEGylated liposomes, and mannosylated liposomes were developed and the cytotoxicity, cellular internalization, immunostimulatory activity, targeting efficiency, antitumor efficacy, and mechanism were evaluated in vitro and in vivo.

Results: All liposomes displayed an ideal particle size, good biocompatibility, and controlled release behavior. Mannosylated liposomes exhibited superior in vitro cellular internalization and tumor spheroid penetration with the aid of the mannose receptor-mediated TAMs-targeting effects. In particular, mannosylated liposomes promoted the polarization of both M0 and M2 to the M1 phenotype by enhancing the expression ratio of CD86/CD206 in vitro. Of note, mannosylated liposomes could inhibit G422 glioma tumor growth, which may be attributed to the polarization of TAMs, as evidenced by the reduction in expression level of the TAMs surface marker.

Conclusion: These results indicate the potential value of mannosylated liposomes in the design of a rational delivery system to enhance the antitumor immune efficacy of immunomodulators by inducing a shift from the M2 to the M1 phenotype.

Keywords: liposomes, cancer immunotherapy, tumor-associated macrophages, mannose receptor, drug delivery

\section{Introduction}

The tumor microenvironment is composed of proliferating tumor cells, tumor stroma, blood and lymphatic vessels, and infiltrating immune cells. ${ }^{1}$ Macrophages are the dominant population of infiltrating immune cells, and are generally termed as tumor-associated macrophages (TAMs). ${ }^{2}$ Accumulating evidence from preclinical and clinical studies indicates that TAMs generally express an M2-like phenotype and play diverse roles in the progression and metastasis of tumors. ${ }^{3,4}$ Particularly, they secrete a plethora of tumor-promoting cytokines and growth 
factors to accelerate tumor growth, angiogenesis, progression and metastasis, and immune suppression. ${ }^{2,5,6}$ The elevated population of TAMs is associated with poor overall prognosis and therapeutic failure in patients with various cancers, ${ }^{7-10}$ suggesting their potential value as a target in cancer immunotherapy. Therefore, strategies that target TAMs, including depletion of TAMs or polarization of the tumor-promoting M2 subtype to the tumorsuppressing M1 subtype, may enhance the efficacy of antitumor therapy. 6,11

With advances in nanomedicine, the development of sophisticated nanocarriers that are functionalized by specific receptors on TAMs is a feasible TAMs-targeting strategy. A number of recent experimental studies have reported the utility of TAMs-targeted nanocarriers loaded with various immunomodulators to inhibit tumor growth and metastasis with satisfactory outcomes in breast cancer, ${ }^{12-14}$ melanoma, ${ }^{15}$ lung cancer, ${ }^{16}$ pancreatic cancer, ${ }^{17}$ glioblastoma, ${ }^{18}$ and sarcoma. ${ }^{19}$ It is well known that macrophages are predominantly responsible for internalizing engineered nanocarriers and then undergo polarization or reprogramming in response to signals, which leads to the expression of various cell surface receptors for cellular uptake as well as cytokine and chemokine secretion. ${ }^{20,21}$ Therefore, the intrinsic impact of drug-free nanocarriers on macrophage physiology is one of the most critical issues in nanomedicine for promoting effective immunotherapy.

Previous studies have shown that a variety of engineered drug-free nanoparticles, such as iron oxide nanoparticles, ${ }^{22}$ noble metal (gold and silver) nanoparticles, ${ }^{23}$ polystyrene nanoparticles, ${ }^{24}$ and glycocalyx-mimicking nanoparticles, ${ }^{25}$ could induce polarization of TAMs from M2 to M1 in the tumor microenvironment, indicating a probable role for nanoparticles as a tool in selective reprogramming of $\mathrm{M} 1 /$ M2 polarization. Despite many studies specifying the intrinsic impact of drug-free nanoparticles on macrophage polarization, very little attention has been placed on the investigation of the interaction between liposomes and macrophages at the cellular level. Given the increasing importance of liposome-based immunotherapy for the treatment of tumors, the cytotoxicity, cellular internalization level and mechanism, intracellular trafficking, and immune response of drug-free liposomes to macrophages warrant particular attention.

The aim of the present study was to gain insight into the interactions, including the cellular internalization process and immune response, between drug-free liposomes and macrophages. Surface functionalization of nanocarriers with specific ligands has an important effect on the interactions between nanocarriers and biological targets. ${ }^{21,24,26,27}$ As TAMs abundantly express mannose receptors (MRs), ${ }^{28}$ mannose-decorated liposomes, conventional bare liposomes, and PEGylated liposomes were developed and evaluated. The specific development of immune modulators targeting TAMs would be of significant interest if the distinct interaction between the macrophage membrane and the liposomes, as well as the subsequent uptake mechanisms, were fully understood.

\section{Material and methods Materials}

Soybean phosphatidylcholine (S100) was purchased from Lipoid GmbH (Ludwigshafen, Germany). Cholesterol, 1,2-distearoyl-sn-glycero-3-phosphoethanolamine- N-[amino (polyethylene glycol)-2000] (DSPE-PEG 2000 ) and DSPE$\mathrm{PEG}_{2000}$-N-hydroxysuccinimide ester (NHS) were purchased from A.V.T. Pharmaceutical Co., Ltd. (Shanghai, China). 4-Aminophenyl $\alpha$-D-mannopyranoside was obtained from J\&K Scientific Ltd. (Beijing, China). Coumarin-6 was purchased from Sigma-Aldrich (Saint Louis, MO, USA). 4',6-Diamidino-2-phenylindole (DAPI) and LysoTracker Red were purchased from Beyotime (Haimen, Jiangsu, China). APC-CD86 and PE-CD206 were supplied by BioLegend (San Diego, CA, USA). The primary antibody against CD206 was obtained from Abcam (Cambridge, MA, USA). The primers of $\mathrm{Arg} 1, \mathrm{Mgl}$, and $\mathrm{Ym} 1$ were obtained from Invitrogen (Carlsbad, CA, USA). Recombinant murine interleukin-4 (IL-4) was supplied by PeproTech (Rocky Hill, NJ, USA). Dulbecco's Modified Eagle's Medium (DMEM) and fetal bovine serum (FBS) were purchased from Thermo Fisher Scientific Inc. (Waltham, MA, USA). Temozolomide capsules were purchased from Jiangsu Tasly Diyi Pharmaceutical Co., Ltd. (Huai'an, Jiangsu, China). All other organic reagents were of analytical grade and purchased from Sinopharm Chemical Reagent Co., Ltd. (Shanghai, China).

\section{Cell culture}

The RAW264.7 murine macrophage cell line was obtained from the Cell Resource Center, Peking Union Medical College (Beijing, China). The 4T1 murine breast adenocarcinoma cell line and G422 murine glioma cell line were kind gifts from Prof. Zhonggao Gao and Prof. Xiaoguang Chen, respectively (Institute of Materia Medica, Peking 
Union Medical College, Beijing, China). RAW264.7, 4T1, and G422 cells were cultured in DMEM supplemented with $10 \%$ FBS, $100 \mathrm{U} / \mathrm{mL}$ penicillin, and $100 \mu \mathrm{g} / \mathrm{mL}$ streptomycin in a humidified atmosphere of $5 \% \mathrm{CO}_{2}$ at $37{ }^{\circ} \mathrm{C}$.

\section{Synthesis of DSPE-PEG 2000 -mannose}

$\mathrm{DSPE}_{-\mathrm{PEG}_{2000} \text {-Mannose (DSPE-PEG }} \mathrm{P}_{200}$-Man) was synthesized in a reaction by covalent binding with DSPE-PEG ${ }_{2000^{-}}$ NHS and 4-Aminophenyl $\alpha$-D-mannopyranoside (Figure 1A). ${ }^{29} \quad$ Briefly, $0.04 \quad$ mol 4-Aminophenyl $\alpha$ D-mannopyranoside was dissolved in $500 \mu \mathrm{L}$ of anhydrous dimethylsulfoxide (DMSO) for $30 \mathrm{~min}$, followed by the addition of 0.02 mol DSPE-PEG 2000 -NHS and stirring at $30^{\circ} \mathrm{C}$ for $48 \mathrm{~h}$. The reaction mixture was purified by dialysis using a membrane with a molecular weight cutoff (MWCO) of 1000 Da (Spectrum Laboratories, Rancho Dominguez, CA, USA) for $48 \mathrm{~h}$. The product was freeze-dried, and the chemical structure of DSPE-PEG 2000 -Man was characterized by ${ }^{1} \mathrm{H}$ nuclear magnetic resonance (NMR).

\section{Preparation and characterization of liposomes}

Liposomes were prepared by the thin film hydration method. Lipids, including S100, cholesterol, DSPE-PEG 2000 , and DSPE-PEG ${ }_{2000}$-Man were dissolved in chloroform at a molar ratio of 65:30:3:2, and the organic solvent was removed by rotary evaporation under vacuum at $40{ }^{\circ} \mathrm{C}$ to form a thin film layer. The lipid film was hydrated in phosphate buffered saline (PBS; $10 \mathrm{mM}$ and $\mathrm{pH} 7.4$, here and hereafter) at $55^{\circ} \mathrm{C}$ for $1 \mathrm{~h}$, and intermittently sonicated by a probe sonicator at $100 \mathrm{~W}$ for $9 \mathrm{~min}$ in an ice-water bath to obtain mannosylated liposomes (Man-PEG-Lipo). Conventional bare liposomes (Lipo) and PEGylated liposomes (PEG-Lipo) were prepared as described above, except that the molar ratio of lipids varied accordingly (S100:cholesterol of $=70: 30$ for Lipo and S100:cholesterol: DSPE-PEG $_{2000}$ of $=65: 30: 5$ for PEG-Lipo). Liposomes loaded with 1,1-dioctadecyl-3,3,3,3-tetramethylindotricarbocyanine iodide (DiR) or coumarin-6 were prepared with similar procedure except that DiR or coumarin-6 was dissolved in chloroform with lipids.

A
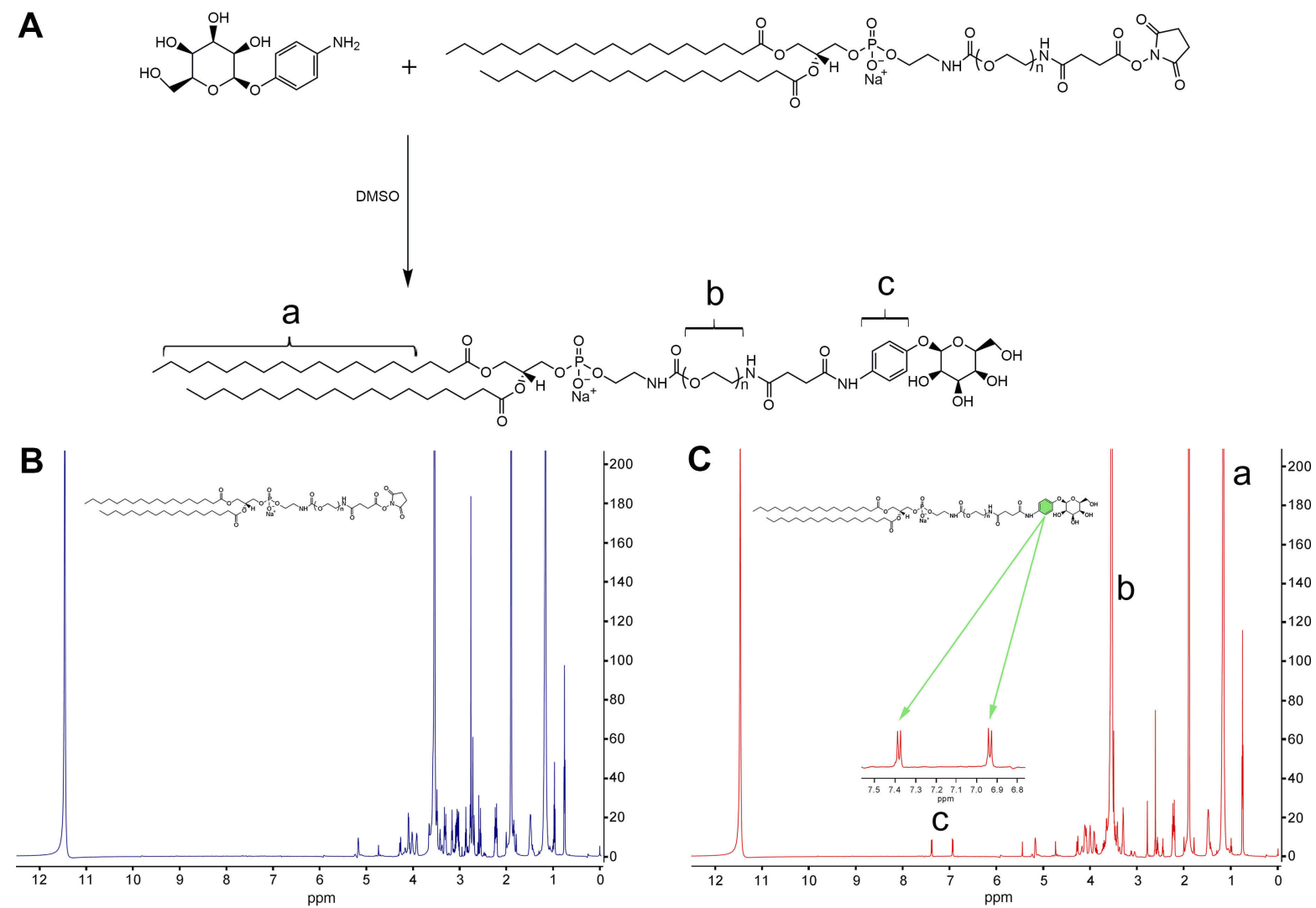

Figure I Synthesis and characterization of DSPE-PEG $2000-$ Mannose. (A) Schematic of synthetic DSPE-PEG $2000-$ Mannose. (B) The 'H-NMR spectrum of DSPE-PEG 2000 . (C) The 'H-NMR spectrum of DSPE-PEG 2000 -Mannose.

Note: a, I.0-1.5 pPm (DSPE); b, 3.5-4.0 PPm (PEG); c, 6.9-7.5 ppm (phenyl group)

Abbreviation: 'H-NMR, 'H-Nuclear Magnetic Resonance. 
The average particle size and size distribution of liposomes were determined by the dynamic light scattering method using Zeta Potential/Particle Sizer NICOMP 380 ZLS (PSS NICOMP, Santa Barbara, CA, USA) after appropriate dilution with double-distilled water. Morphological examination of liposomes was carried out by transmission electron microscopy (TEM) (SU8010; Hitachi, Tokyo, Japan). Briefly, samples were prepared by adding one drop of liposome suspension to a copper grid followed by staining with $2 \%(\mathrm{w} / \mathrm{v})$ phosphotungstic acid. The samples were dried and examined.

\section{In vitro drug release assay}

The in vitro release of coumarin- 6 from liposomes was assayed in $0.5 \%(\mathrm{w} / \mathrm{v})$ Tween-80 in PBS using a dialysis method. The coumarin-6-loaded liposomes were placed in a dialysis bag (MWCO $12 \mathrm{kDa}$ ) and immersed in $100 \mathrm{~mL}$ of release medium at $37{ }^{\circ} \mathrm{C}$ on a shaker $(100 \mathrm{rpm})$. At predetermined timepoints, $1 \mathrm{~mL}$ of release medium was withdrawn and replaced with fresh release medium. The concentration of coumarin- 6 was determined by highperformance liquid chromatography with a fluorescence detector (1260; Agilent Technologies, Santa Clara, CA, USA). Each sample was performed in triplicate.

\section{In vitro cytotoxicity}

The in vitro cytotoxicity of conventional bare liposomes, PEGylated liposomes, and mannosylated liposomes was evaluated in RAW264.7 macrophages using CCK-8 kits (Dojindo Laboratories, Tokyo, Japan). Briefly, RAW264.7 cells were plated at a density of $2 \times 10^{4}$ cells per well in a 96-well plate. After $24 \mathrm{~h}$, the cells were treated with different liposome samples containing various concentrations of lipids $(0,10,50,100,500$, and $1000 \mu \mathrm{g} / \mathrm{mL})$. After $24 \mathrm{~h}$ of incubation, the number of viable cells was determined using CCK-8 kits according to the manufacturer's protocol. Untreated control cells were used as the control and were considered to be $100 \%$ viable.

\section{In vitro cellular uptake}

The cellular uptake index of the different liposomes loaded with coumarin-6 was evaluated by flow cytometry and confocal laser scanning microscopy (CLSM). Briefly, RAW264.7 cells were seeded into 6-well plates at a density of $5 \times 10^{5}$ cells/well and incubated for $24 \mathrm{~h}$. Then, the cells were pre-treated with $20 \mathrm{ng} / \mathrm{mL}$ IL-4 for $24 \mathrm{~h}$ followed by incubation with liposome-encapsulated coumarin- 6 at $37{ }^{\circ} \mathrm{C}$ for $1 \mathrm{~h}$. For CLSM, the cells were rinsed three times with cold PBS, fixed with $4 \%$ paraformaldehyde (PFA), stained with DAPI, and observed by CLSM (FV1000; Olympus, Tokyo, Japan). For flow cytometry analysis, the cells were harvested, washed three times with cold PBS, and then analyzed using a flow cytometer (Accuri C6; BD Biosciences, San Jose, CA, USA).

\section{Three-dimensional co-cultured tumor spheroid penetration}

Three-dimensional (3D) co-cultured multicellular tumor spheroids of tumor cells (4T1 cell line) and macrophages (RAW264.7 cell line) were developed using a liquid-overlay system according to the culture scheme illustrated in Figure 6A. ${ }^{30}$ RAW264.7 cells were stained with $10 \mu \mathrm{g} / \mathrm{mL}$ 1,1'-dioctadecyl-3,3,3',3'-tetramethylindocarbocyanine perchlorate (DiI) prior to spheroid formation. 4T1 cells $\left(1 \times 10^{4}\right.$ cells) and DiI-labeled RAW264.7 cells $\left(0.5 \times 10^{4}\right.$ cells) were mixed with DMEM containing 10\% FBS and 2.5\% Matrigel, seeded in a 96-well round bottom plate (Corning, Corning, NY, USA), and centrifuged at $1000 \times g$ for $10 \mathrm{~min}$. After 3 days, separate groups of co-cultured tumor spheroids were incubated with Lipo, PEG-Lipo, and Man-PEG-Lipo for $8 \mathrm{~h}$. At the end of incubation, the co-cultured tumor spheroids were washed three times with cold PBS, and fixed with 4\% PFA for $1 \mathrm{~h}$ at room temperature. The fluorescence intensity of different depths of the co-cultured tumor spheroids was determined using CLSM.

\section{Cellular internalization mechanism and intracellular trafficking}

To study whether endocytosis was the main pathway for the cellular uptake of Man-PEG-Lipo, RAW264.7 cells were pre-treated with $20 \mathrm{ng} / \mathrm{mL} \mathrm{IL}-4$ for $24 \mathrm{~h}$ and then incubated with Man-PEG-Lipo at $4{ }^{\circ} \mathrm{C}$ instead of the regular $37{ }^{\circ} \mathrm{C}$. Additionally, cells were pre-incubated with different inhibitors, including chlorpromazine $(10 \mu \mathrm{g} / \mathrm{mL}),{ }^{31}$ cytochalasin $\mathrm{D}(10 \mu \mathrm{M}),{ }^{32}$ and methyl- $\beta$-cyclodextrin $(10 \mathrm{mM}),{ }^{31}$ at $37^{\circ} \mathrm{C}$ for $1 \mathrm{~h}$. Then, Man-PEG-Lipo and the different inhibitors at the same concentrations as described were added and incubated at $37{ }^{\circ} \mathrm{C}$ for another $2 \mathrm{~h}$. At the end of incubation, cells were washed, trypsinized, and analyzed by flow cytometry.

To investigate the intracellular fate of Man-PEG-Lipo, RAW264.7 cells were plated on glass bottom dishes in a 6 -well plate at a density of $1 \times 10^{5}$ cells/well and incubated for $24 \mathrm{~h}$ at $37^{\circ} \mathrm{C}$. After pre-treatment with $20 \mathrm{ng} / \mathrm{mL}$ IL-4 for $24 \mathrm{~h}$, the cells were incubated with Man-PEGLipo for $1 \mathrm{~h}$ at $37^{\circ} \mathrm{C}$. At the end of incubation, cells were 
stained with $75 \mathrm{nM}$ LysoTracker Red, fixed with 4\% PFA, and stained with DAPI. Fluorescence images of cells were captured with CLSM.

\section{Tissue biodistribution}

Female BALB/c mice weighing 14-16 g were supplied by Beijing Vital River Laboratory Animal Technology Co., Ltd. (Beijing, China). All animal experiments were approved by the Institutional Animal Care and Use Committee of Peking Union Medical College. The care of laboratory animal and animal experimental operation were performed in accordance with the Beijing Administration Rule of Laboratory Animal. Orthotopic 4T1 breast tumors were generated by injecting $1 \times 10^{5}$ cells into the mammary fat pad of each mouse. When the tumors grew to a median size of $500 \mathrm{~mm}^{3}$, DiR-encapsulated liposomes were injected into the tail vein of each mouse $(0.1 \mathrm{mg} / \mathrm{kg}$ body weight $)$. During the experiments, the whole-body fluorescence images were detected at predetermined times postinjection $(2,4,8,12$, and $24 \mathrm{~h})$ using the In Vivo IVIS spectrum-imaging system (PerkinElmer, Waltham, MA, USA). At the end of experiments, the mice were sacrificed, the tumors and other major organs were surgically collected, and examined by ex vivo imaging.

\section{Macrophage marker expression}

RAW264.7 cells were seeded in 6-well plates at a density of $5 \times 10^{5}$ cells/well and treated with IL-4 $(20 \mathrm{ng} / \mathrm{mL})$ for $24 \mathrm{~h}$ to induce the polarization of RAW264.7 cells to M2 macrophages. RAW264.7 cells (considered as M0 macrophages) or M2 macrophages were treated with the different liposomes $(500 \mu \mathrm{g} / \mathrm{mL})$ for $24 \mathrm{~h}$, followed by incubation with antibodies to two different cell surface markers (CD86 for M1 macrophages and CD206 for M2 macrophages). After incubation and rinsing, the samples were analyzed by flow cytometry.

\section{In vivo antitumor efficacy and safety evaluation}

Female ICR mice (6 8 weeks old) were purchased from SPF (Beijing) Biotechnology Co., Ltd. (Beijing, China). All animal protocols were approved by the institutional animal care and use committee of Peking Union Medical College. Glioma tumor model was established by the subcutaneous injection of $5 \times 10^{5}$ G422 cells to the right flank of each ICR mouse. The tumor-bearing mice were randomly assigned five groups ( $n=7$ per group) the following day after tumor implantation to receive blank liposomes at 47 nmoles of phospholipids/g body weight or vehicle via tail vein injections daily for two weeks as described in the schedule given, according to the treatment scheme illustrated in Figure 10A. The positive control group was orally treated with temozolomide (TMZ) $(50 \mathrm{mg} / \mathrm{kg} / \mathrm{day})$ for 5 days. At the end of the experiments, all mice were sacrificed and the tumors were collected, photographed, weighed and further analyzed by quantitative real-time PCR for Arg1, Mgl2, and Ym1 mRNA expression and western blot for CD206 expression. ${ }^{33}$ The antitumor efficacy was evaluated by calculating tumor growth inhibition ratio (TGI\%) according to the formula shown below:

$$
\mathrm{TGI} \%=\left(1-\frac{W_{\text {test }}}{W_{\text {vehicle }}}\right) \times 100
$$

$\mathrm{W}_{\text {test }}$ and $\mathrm{W}_{\text {vehicle }}$ represent the tumor weight of the treatment group and the vehicle group, respectively.

\section{Statistical analysis}

All data subjected to statistical analysis were obtained from at least three parallel experiments, and the results are expressed as mean \pm standard deviation (SD). The statistical analysis was performed by unpaired two-tailed Student's $t$-test for two groups, and one-way ANOVA for multiple groups using GraphPad Prism version 7.00 for Windows (GraphPad Software, La Jolla, CA, USA). A $p$-value $\leq 0.05$ was considered statistically significant.

\section{Results Preparation and characterization of liposomes}

The ${ }^{1} \mathrm{H}-\mathrm{NMR}$ spectrum of DSPE-PEG 2000 and DSPE-PEG $2000^{-M a n}$ are shown in Figure $1 \mathrm{~B}$ and $\mathrm{C}$. The peaks at 6.9-7.5 ppm represented the phenyl group in 4-Aminophenyl $\alpha$-D-mannopyranoside, indicating that the NHS group had reacted with the amino group. The peaks of ${ }^{1} \mathrm{H}-\mathrm{NMR}$ spectrum were assigned to chemical structures as follows: 6.9-7.5 ppm (phenyl group), 3.5-4.0 ppm (PEG), and 1.0-1.5 ppm (DSPE). The reaction yield of DSPE-PEG 2000 -Man synthesis was approximately $81.4 \%$.

The mean particle size of Lipo, PEG-Lipo, and ManPEG-Lipo was 133.9 nm, 105.7 nm, and $109.8 \mathrm{~nm}$, respectively (Figure 2). All liposomes had polydispersity index (PDI) values below 0.3 , demonstrating that the size distribution of all liposomes was narrow. TEM examination of Lipo, PEG-Lipo, and Man-PEG-Lipo samples revealed 

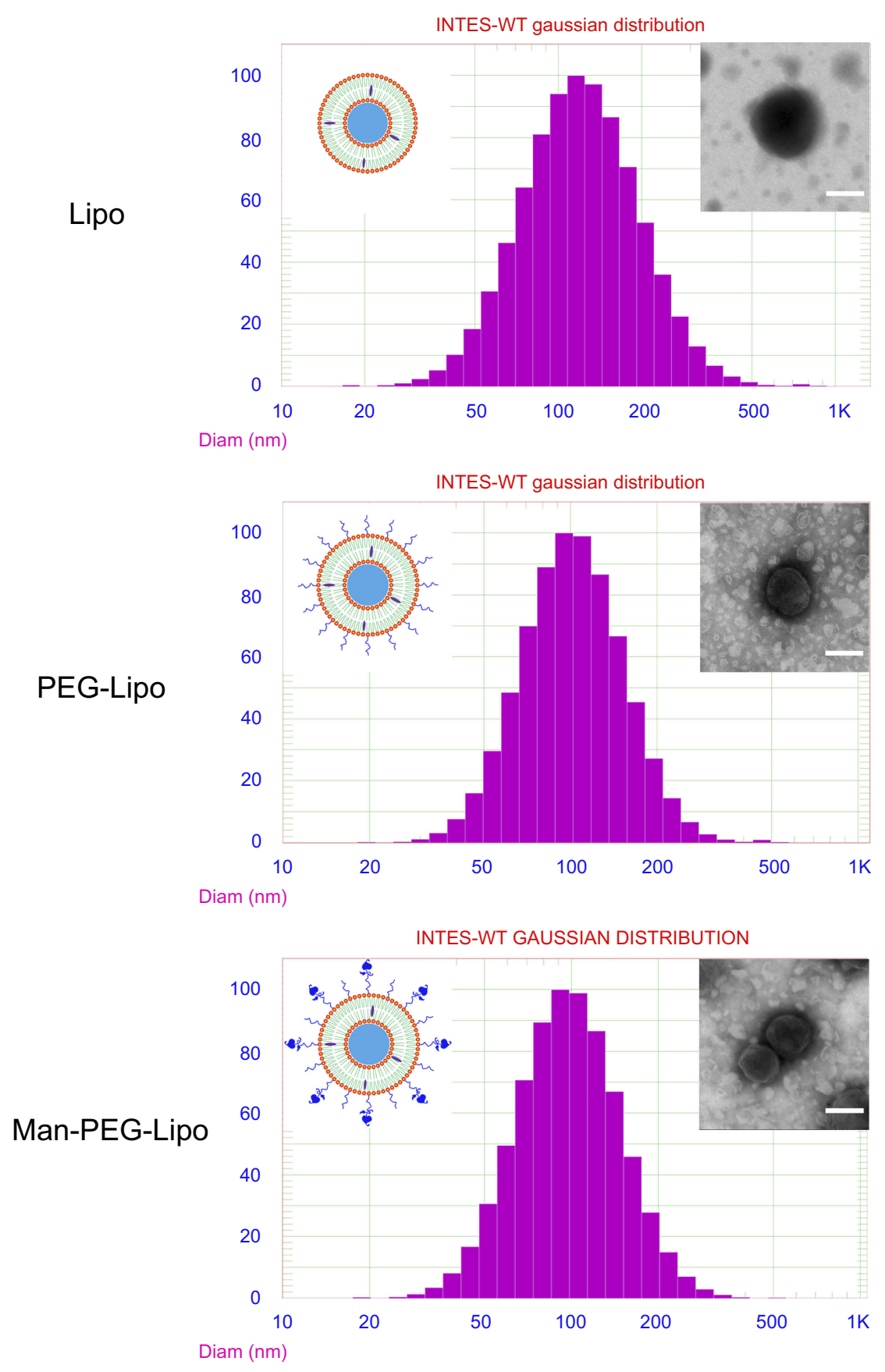

Figure 2 Particle size distribution and morphology of Lipo, PEG-Lipo, and Man-PEG-Lipo. Scale bar $=100 \mathrm{~nm}$. Abbreviations: Lipo, liposomes; PEG-Lipo, PEGylated liposomes; Man-PEG-Lipo, mannosylated liposomes.

that the three kinds of liposomes were spherical and regular in shape (Figure 2).

\section{In vitro release}

As shown in Figure 3, the in vitro release of coumarin-6 from Lipo, PEG-Lipo, and Man-PEG-Lipo displayed similar patterns of gradual increase within $72 \mathrm{~h}$ in the release medium, suggesting that all three liposomes had a sustained release profile.

\section{In vitro cytotoxicity}

In vitro cytotoxicity of Lipo, PEG-Lipo, and Man-PEGLipo was evaluated on RAW264.7 cells using CCK-8 kits. Incubation of the various concentrations of the three liposomes produced negligible RAW264.7 cytotoxicity (Figure 4), indicating the excellent biocompatibility of the liposomes with macrophages. Interestingly, lipid concentrations exceeding $500 \mu \mathrm{g} / \mathrm{mL}$ produced a higher cell survival rate compared with the control, indicating that 


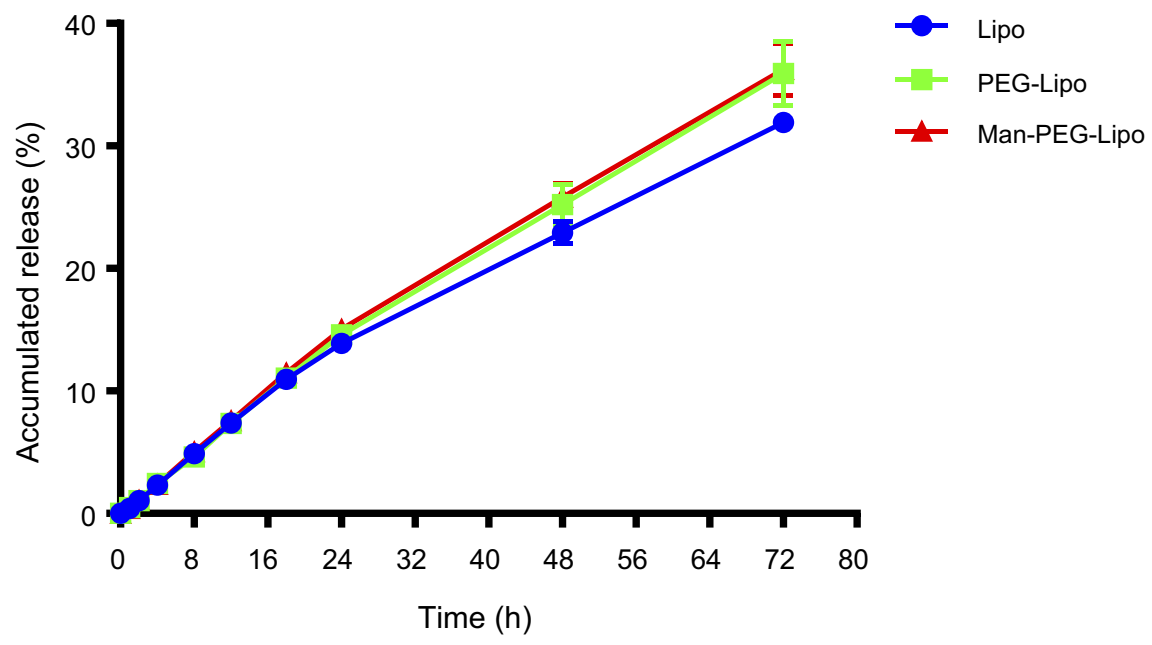

Figure 3 In vitro release profile of liposomes in PBS $(\mathrm{pH} 7.4)$ containing $0.5 \%(w / v)$ Tween 80 . Each value represents the mean \pm SD $(n=3)$. Abbreviations: Lipo, liposomes; PEG-Lipo, PEGylated liposomes; Man-PEG-Lipo, mannosylated liposomes; PBS, phosphate buffered saline.

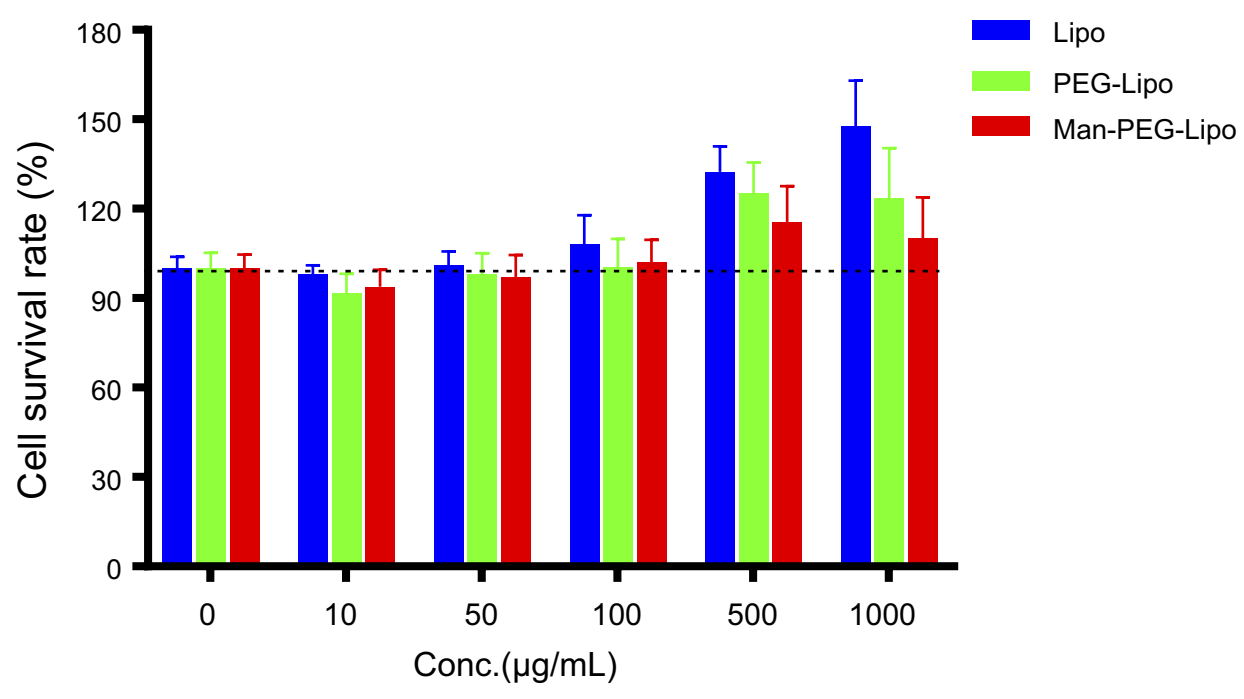

Figure 4 In vitro cytotoxicity of liposomes in RAW264.7 cells at $24 \mathrm{~h}$. Each value represents the mean \pm SD $(n=6)$.

Abbreviations: Lipo, liposomes; PEG-Lipo, PEGylated liposomes; Man-PEG-Lipo, mannosylated liposomes.

high lipid levels of all three liposomes enhanced macrophage proliferation.

\section{In vitro cellular uptake}

The cellular uptake index of coumarin-6-encapsulated liposomes by M2 macrophages was determined qualitatively by CLSM and quantitatively by flow cytometry. The green fluorescence was dispersed in the surrounding nuclear membrane (Figure 5A), demonstrating that the liposomes were able to effectively deliver coumarin- 6 to the cytoplasm of M2 macrophages. It is worth noting that the fluorescence intensity was clearly enhanced following $1 \mathrm{~h}$ incubation with Man-PEG-Lipo as compared with Lipo and
PEG-Lipo. Additionally, the uptake of Man-PEG-Lipo was significantly increased in M2 macrophages compared with that in RAW264.7 cells untreated with IL-4. To further evaluate the cellular uptake efficiency of the different liposomes, the mean fluorescence intensity (MFI) was determined by flow cytometry. Consistent with the CLSM results, the highest MFI was evident in cells exposed to Man-PEG-Lipo, compared with Lipo and PEG-Lipo (Figure 5B and C).

\section{D co-cultured tumor spheroid penetration}

Given the critical role TAMs play in tumors, the development of in vitro 3D co-cultured tumor spheroids, which 


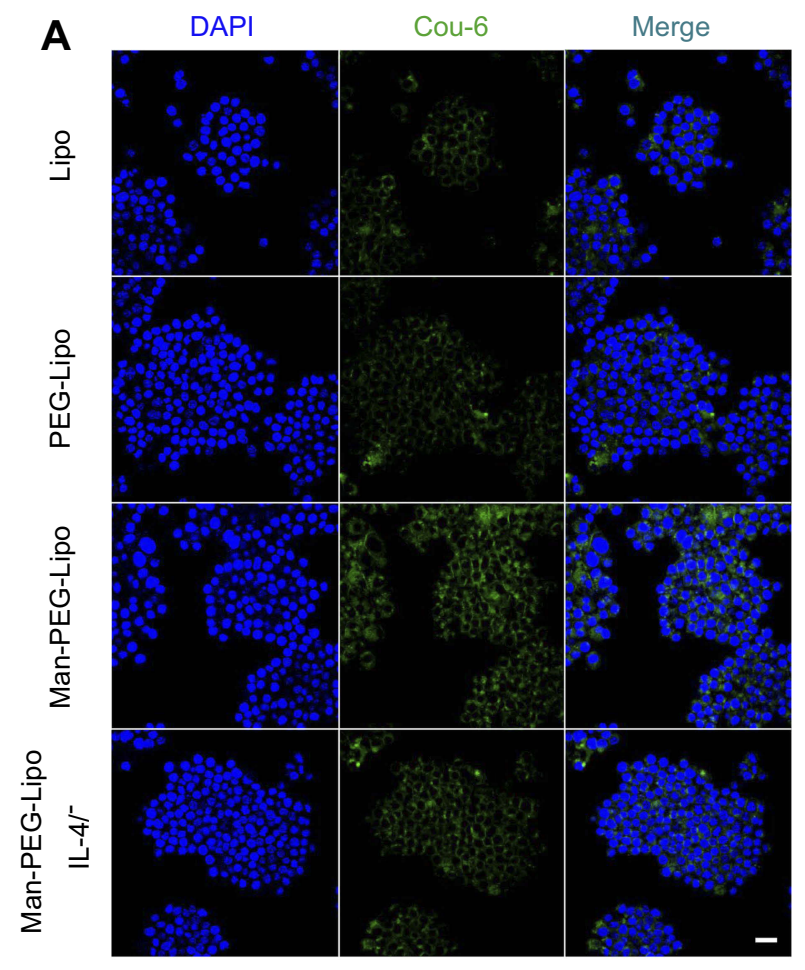

B

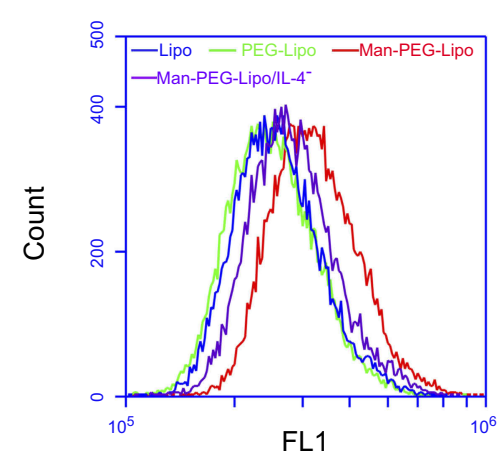

C

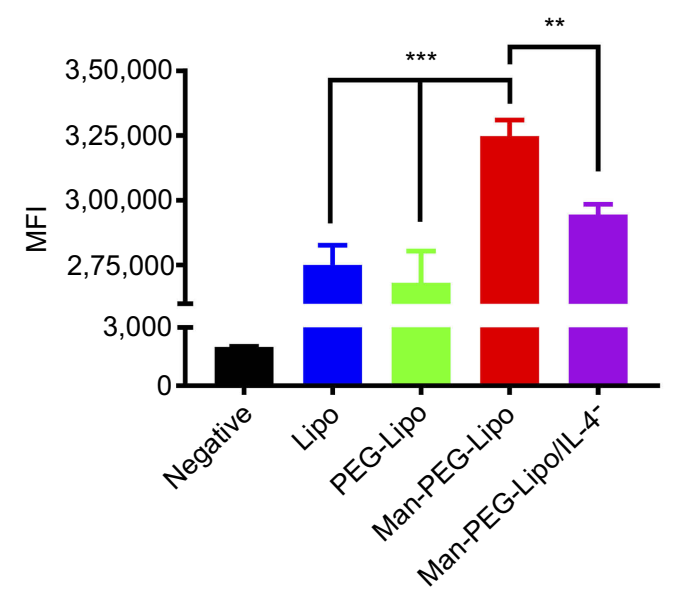

Figure 5 In vitro cellular uptake of coumarin-6-loaded liposomes in RAW264.7 cells or IL-4-conditioned RAW264.7 cells. (A) CLSM images of cellular uptake. (B and $\mathbf{C}$ ) Mean fluorescence intensity was determined by flow cytometry. Scale bar $=20 \mu \mathrm{m}$. Each value represents the mean \pm SD $(n=3)$. ** $p<0.0 \mathrm{I}$ and $* * * p<0.00 \mathrm{I}$. Abbreviations: Lipo, liposomes; PEG-Lipo, PEGylated liposomes; Man-PEGLipo, mannosylated liposomes; IL-4, Interleukin-4; CLSM, confocal laser scanning microscopy. recapitulate the interplay between TAMs and cancer cells, is of significant basic and clinical interest. ${ }^{30}$ To this end, $3 \mathrm{D}$ co-cultured tumor spheroids of $4 \mathrm{~T} 1$ and RAW264.7 cells were used to evaluate the penetration efficiency of the liposomes. Morphological analysis by scanning electron microscopy (SEM) demonstrated that the cells formed tightly packed, rounded spheroids (Figure 6B). The CLSM image showed that DiI-labeled RAW264.7 cells (red fluorescence) distributed homogeneously throughout the spheroid (Figure 6B). When co-cultured tumor spheroids were incubated with the coumarin-6-loaded liposomes for $8 \mathrm{~h}$, Man-PEG-Lipo penetrated more deeply and was distributed more extensively in tumor spheroids than Lipo and PEG-Lipo, indicating that mannose receptor led to higher penetration efficiency of Man-PEG-Lipo in co-cultured tumor spheroids (Figure 6C).

\section{Cellular internalization mechanism and intracellular trafficking}

To determine the cellular internalization mechanism of ManPEG-Lipo, biochemical inhibitors, including chlorpromazine, cytochalasin $\mathrm{D}$, and methyl- $\beta$-cyclodextrin, were selected to inhibit clathrin-, macropinocytosis-, and caveolinmediated endocytosis, respectively. ${ }^{31,32}$ After incubation at $4{ }^{\circ} \mathrm{C}$, the cellular uptake of Man-PEG-Lipo decreased dramatically as compared with the control $\left(37^{\circ} \mathrm{C}\right)$, indicating that the endocytosis was energy-dependent (Figure 7A). When cells were pre-treated with chlorpromazine, cytochalasin $\mathrm{D}$, or methyl- $\beta$-cyclodextrin, the reduction of cellular uptake ratio was $92.5 \%, 97.2 \%$, and $10.0 \%$, respectively, demonstrating that the internalization of Man-PEG-Lipo primarily relied on caveolae-mediated endocytosis pathways.

The intracellular trafficking of Man-PEG-Lipo after internalization was investigated by CLSM. After incubation, coumarin-6 (green fluorescence) was dispersed in the cytoplasm and co-localized with lysosomes (red fluorescence), indicating that Man-PEG-Lipo was first localized in lysosomes after internalization by macrophages (Figure 7B).

\section{Tissue biodistribution}

The tumor fluorescence in DiR-Man-PEG-Lipo-treated mice was significantly higher than that in the DiR-DMSO-, DiRLipo-, and DiR-PEG-Lipo-treated mice at all time points following the injection of the liposomes (Figure 8A). The ex vivo fluorescent image from the excised tumors also clearly showed higher fluorescence from the DiR-Man-PEG-Lipotreated groups compared to the DiR-DMSO-, DiR-Lipo-, 


\section{A}

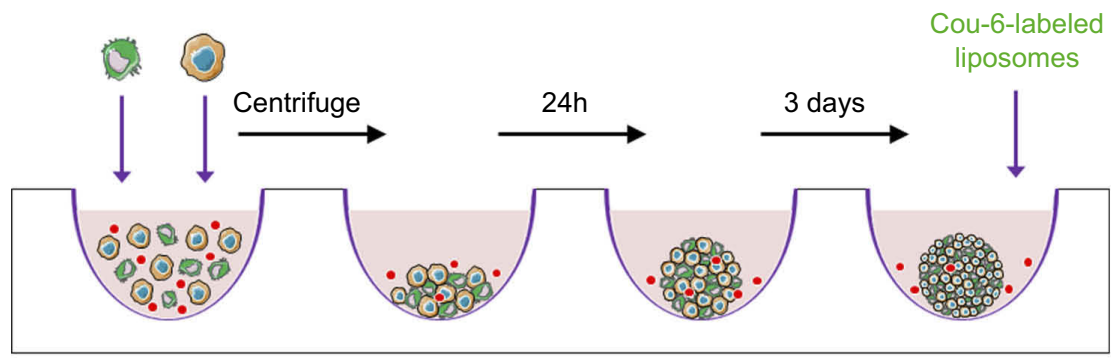

Macrophages
Tumor cells
Matrigel
B

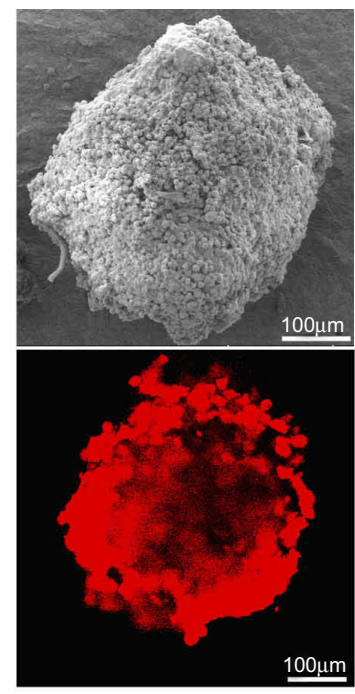

C

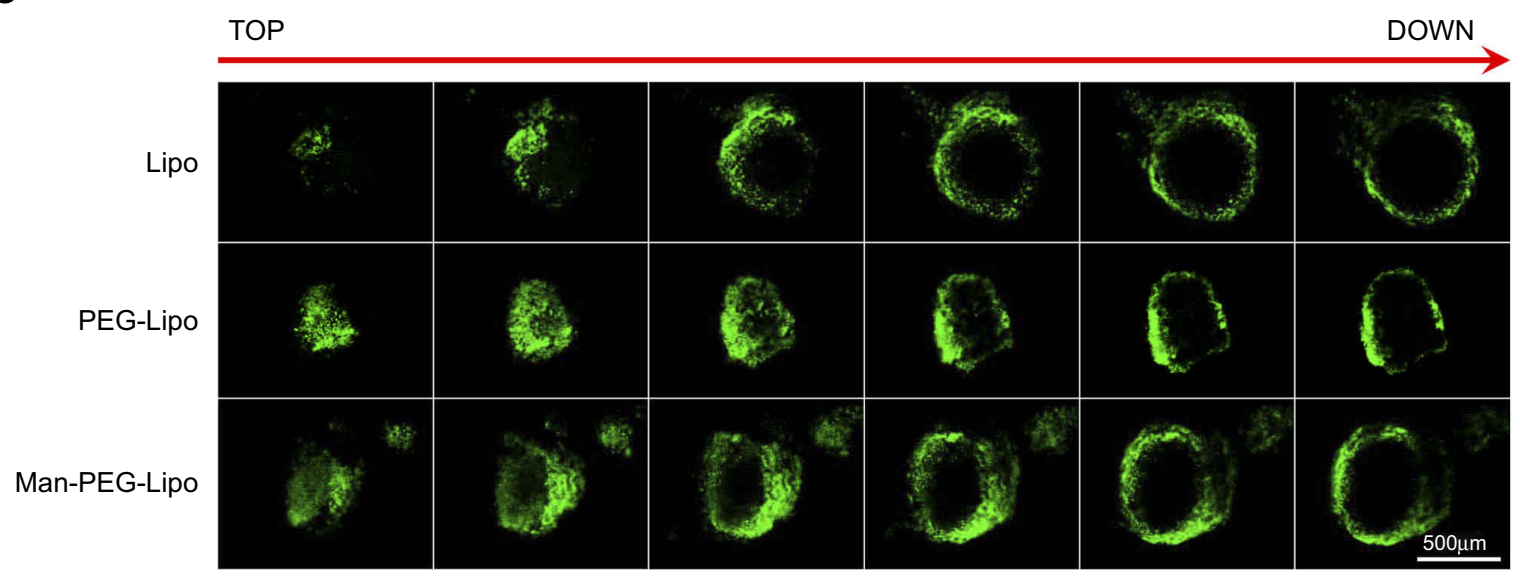

Figure 6 3D co-cultured multicellular tumor spheroids penetration. (A) The culture and penetration experiment procedure of 3D co-cultured multicellular tumor spheroids. (B) Images of 3D co-culture multicellular tumor spheroids by SEM and CLSM. RAW264.7 cells were labeled with Dil. Scale bar $=100 \mu$ m. (C) Penetration of coumarin-6-loaded liposomes in 3D co-cultured multicellular tumor spheroids. Scale bar $=500 \mu \mathrm{m}$.

Abbreviations: Lipo, liposomes; PEG-Lipo, PEGylated liposomes; Man-PEG-Lipo, mannosylated liposomes; 3D, three-dimensional; SEM, scanning electron microscope; CLSM, confocal laser scanning microscopy.

and DiR-PEG-Lipo-treated groups (Figure 8B). Additionally, the 3D reconstruction of DiR-Man-PEG-Lipo-treated mice was performed to observe the overall distribution of fluorescence in the tumor. As shown in Figure 8C, the fluorescence was primarily found around the tumor and partially observed in the center of the tumor, indicating that Man-PEG-Lipo may be used as an effective TAMs-targeting carrier by which to deliver immunomodulators to tumor tissues.

It is of significance to explore the accumulation of mannosylated liposomes in the liver since Kupffer cells distributed there have been identified to express mannose receptor. ${ }^{34,35}$ As shown in Figure 8B and S1A, mice treated with mannosylated liposomes displayed elevated fluorescent signals in livers compared with PEGylated liposomes, and the fluorescence in spleens was comparable between the two groups. The enhanced liver accumulation might be due to the mannose receptor expression in liver Kupffer cells, which is in accordance with previous related studies. ${ }^{36,37}$ Although mannosylated liposomes could enhance the liver accumulation, both the tumor-to-liver ratio and tumor-tospleen ratio of the mannosylated liposome-treated group were higher than PEGylated liposomes (Figure S1B), indicating that mannosylated liposomes effectively accumulated in tumors.

The collective findings demonstrated the specific and efficient tumor targeting behavior of Man-PEG-Lipo, which may be attributed to the long circulation and the TAMs-targeting ability of Man-PEG-Lipo. 
A

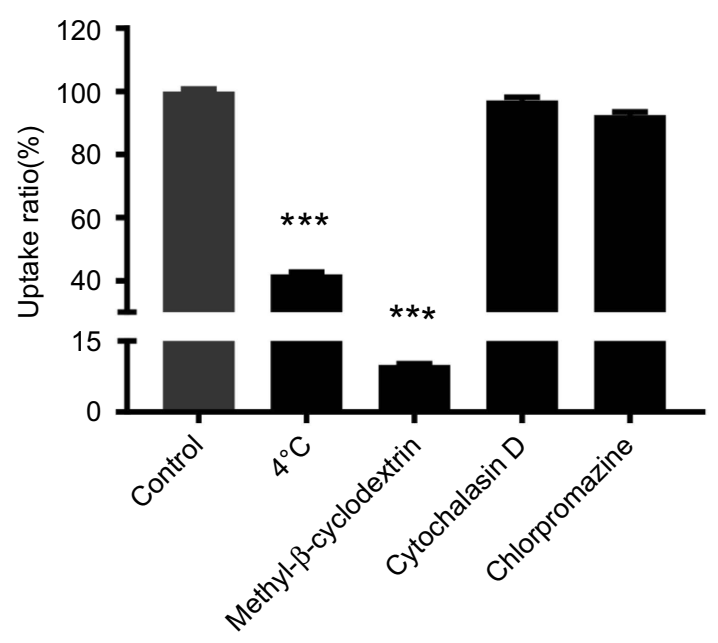

B

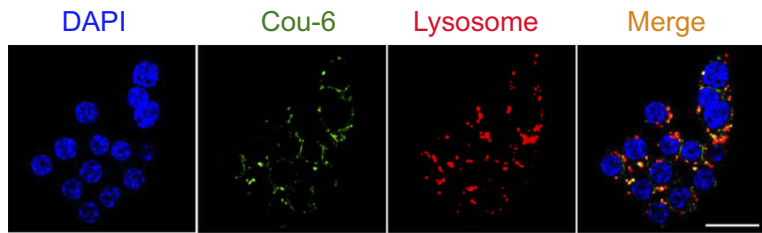

Figure 7 Cellular internalization pathway and intracellular trafficking of Man-PEGLipo. (A) Effects of endocytosis inhibitors on the cellular uptake of Man-PEG-Lipo. (B) Intracellular trafficking of Man-PEG-Lipo. Scale bar $=20 \mu \mathrm{m}$. Each value represents the mean $\pm S D(n=3)$. $* * * p<0.00$ l compared with the control group. Abbreviation: Man-PEG-Lipo, mannosylated liposomes.

\section{The polarization of $M 2$ macrophages in vitro}

To investigate the immune response of liposomes to macrophages, the surface receptor expression was determined by flow cytometry. Differential receptor expression is a critical feature of polarized macrophages. The M1 phenotype is characterized by elevated expression of CD86, whereas M2 macrophages typically expressed CD206. ${ }^{38,39}$ As shown in Figure 9A, the expression ratio of CD86/CD206 was decreased in RAW264.7 cells (considered as the M0 phenotype) treated with IL-4, indicating that IL-4 could induce the polarization of M0 macrophages to the M2 phenotype. Interestingly, the expression ratio of CD86/CD206 in M0 macrophages incubated with ManPEG-Lipo was elevated as compared with control, while Lipo and PEG-Lipo retained the comparative expression ratio, suggesting that Man-PEG-Lipo could promote the polarization of the M0 to the M1 phenotype (Figure 9B). It is worth noting that the upregulation of the CD86/CD206 expression ratio was induced by Man-PEG-Lipo when IL4-conditioned M2 macrophages were treated with ManPEG-Lipo (Figure 9C). These results demonstrated that
Man-PEG-Lipo not only could facilitate polarization of M0 to M1 phenotype, but also had a significant effect on resetting M2 macrophages toward the M1 phenotype.

\section{In vivo antitumor efficacy and safety evaluation}

To investigate whether blank liposomes exposure would impact tumor growth in vivo, a murine G422 glioma tumor model was established and treated with three kinds of blank liposomes daily for 14 days. The dosage of blank liposome was set as 47 nmoles phospholipids/g body weight as previously reported. ${ }^{40}$ As shown in Figure 10D and E, positive drug TMZ dramatically inhibited tumor growth and possessed the lowest tumor weight, with a tumor growth inhibition ratio (TGI\%) of 78.6\%, demonstrating that the murine G422 glioma tumor model was established successfully. Compared to the modest tumor inhibition of blank Lipo (TGI\%, 28.5\%) and PEG-Lipo (TGI\%, 20.0\%), blank Man-PEG-Lipo exhibited superior antitumor efficacy with the highest TGI\% of $40.7 \%$ among the groups treated with three kinds of blank liposomes. The photos and weight of excised tumors showed a similar tendency (Figure 10B and D). These results indicated that blank liposomes could inhibit G422 glioma tumor growth to varying degrees, and Man-PEG-Lipo possessed the greatest potential, which may be attributed to the promotional polarization of M2 macrophages.

In addition, the weight of all groups of mice increased at the end of the experiment compared with that before treatment (Figure 10C), indicating that the three kinds of blank liposomes had the good compatibility in vivo and could be used as a safe drug delivery carrier for future application.

\section{The antitumor mechanism of Man-PEG- Lipo in vivo}

Given that TAMs could promote the progression of tumors and were the primary cells that internalized liposomes in the tumor microenvironment, ${ }^{41}$ it is meaningful to explore whether the tumor growth inhibition of Man-PEG-Lipo is associated with the polarization of TAMs in tumor tissue. The expression level of TAMs surface marker CD206 was significantly reduced in all liposome-treated groups compared to the vehicle-treated group (Figure 11A). However, the positive drug TMZ could not regulate the expression of CD206, indicating that the polarization of TAMs may be not involved in the cytotoxic agent TMZ-mediated inhibition of the tumor growth. It is remarkable that Man-PEGLipo induced the most pronounced reduction in CD206 
A

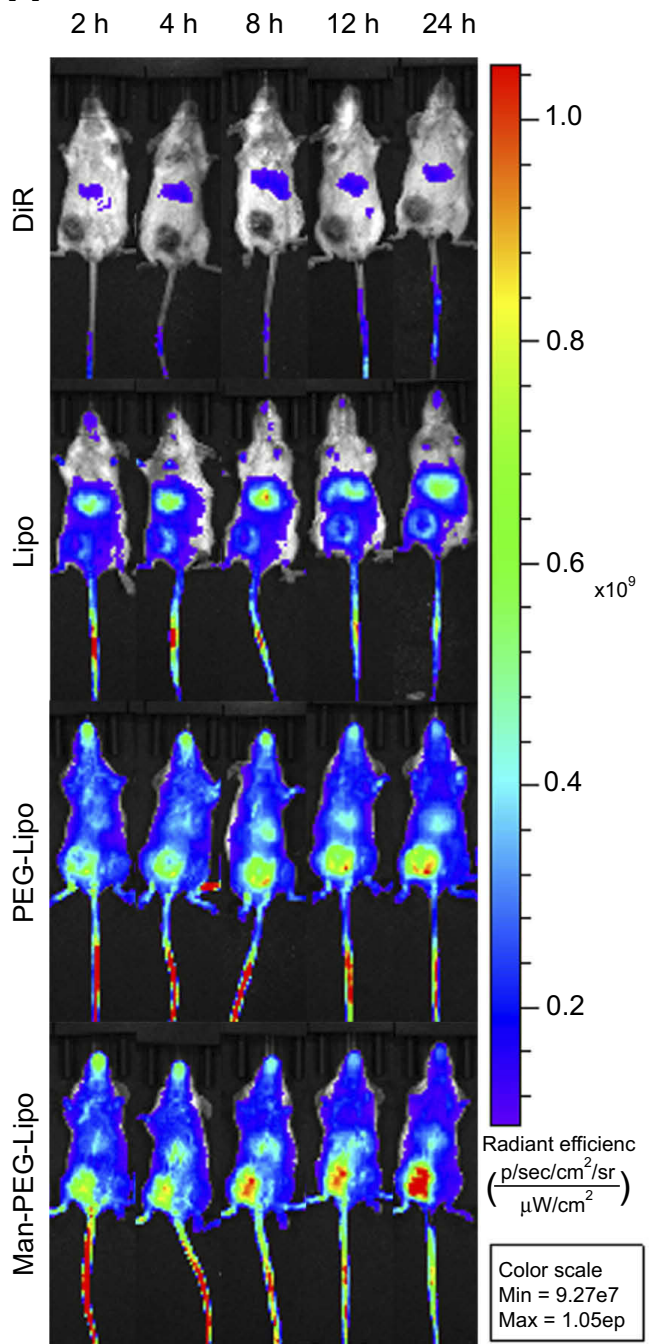

B

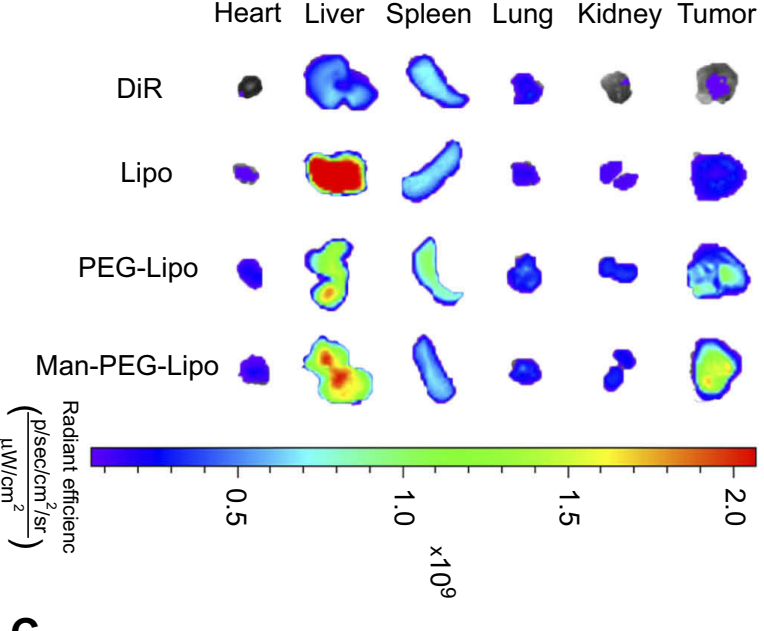

C

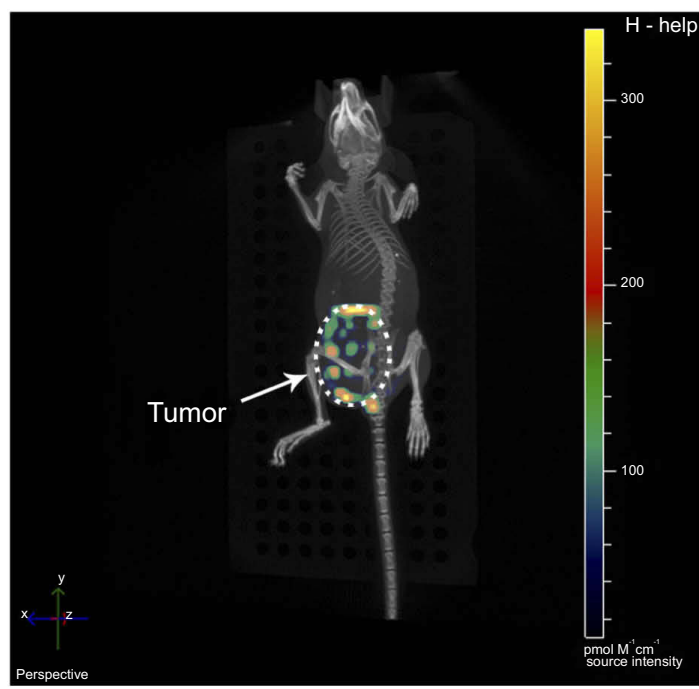

Figure 8 In vivo biodistribution of DiR-loaded liposomes. (A) In vivo fluorescence images of BALB/c mice bearing 4TI after tail vein injection of DiR-loaded liposomes. (B) Ex vivo fluorescence images of excised organs and tumors at $24 \mathrm{~h}$ post-injection of DiR-loaded liposomes. (C) 3D reconstruction of in vivo imaging of DiR-Man-PEG-Lipotreated mice.

Abbreviations: Lipo, liposomes; PEG-Lipo, PEGylated liposomes; DiR, I, I-dioctadecyl-3,3,3,3-tetramethylindotricarbocyanine iodide; 3D, three-dimensional; Man-PEGLipo, mannosylated liposomes.

expression compared with PEG-Lipo and Lipo, which was consistent with the results of the in vivo antitumor efficacy. Due to the polarization of macrophages accompanied by changes in gene expression, the expression of TAMs marker genes in tumor tissue, including $\mathrm{Arg} 1, \mathrm{Mgl} 2$ and $\mathrm{Ym} 1$, was assessed by quantitative real-time PCR. The mRNA expression level of $\mathrm{Mgl} 2$ and $\mathrm{Ym} 1$ were significantly decreased upon treatment with Man-PEG-Lipo compared to the vehicle-treated group (Figure 11C and D). Although there was no statistical difference between the two groups, the $\operatorname{Arg} 1$ mRNA expression level of Man-PEG-Lipo-treated group had a tendency to decrease as compared to that of the vehicle-treated group (Figure 11B). Taken together, these results clearly demonstrated that Man-PEG-Lipo could effectively reduce the polarization of TAMs in vivo. Therefore, the tumor growth inhibition of Man-PEG-Lipo may be attributed to the impact of Man-PEG-Lipo on the polarization of TAMs (Scheme 1).

\section{Discussion}

Cancer immunotherapy holds great potential for the treatment of tumors, and increasing evidence indicates that the stratification of the tumor microenvironment according to tumor-infiltrating lymphocytes is crucial in optimizing cancer immunotherapies. ${ }^{42}$ As the major constituent of tumor-infiltrating lymphocytes, TAMs are generally driven by tumor-derived and $\mathrm{T}$ cell-derived cytokines to acquire a polarized M2 phenotype, and subsequently facilitate 
A

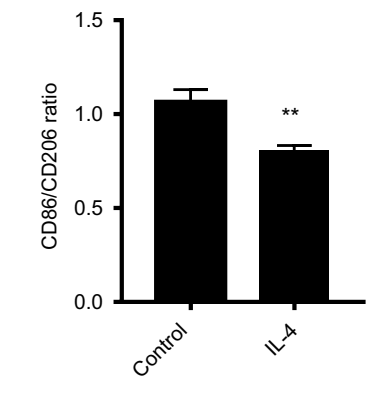

B

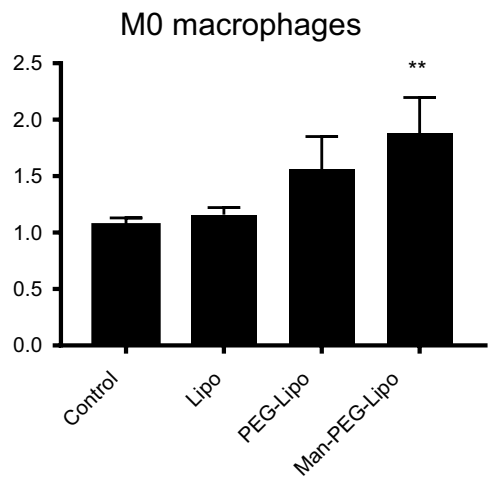

C

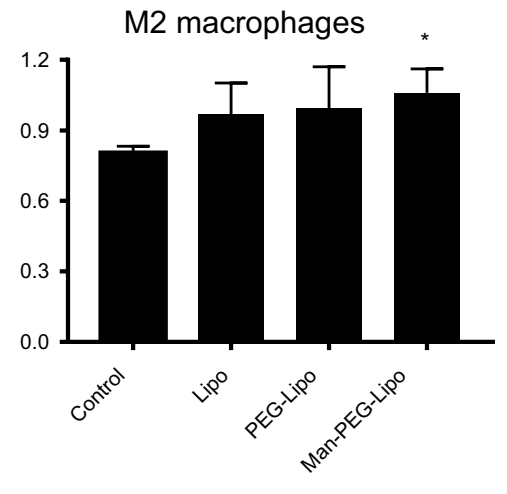

$-$

- $\quad-\quad-$

$+\quad+\quad+\quad+$

Figure 9 Receptor expression ratio of RAW264.7 cells (M0 macrophages) or IL-4-conditioned RAW264.7 cells (M2 macrophages) after incubation with liposomes for 24 h. (A) M0 macrophages were treated with IL-4 for $24 \mathrm{~h}$. (B) M0 macrophages were treated with liposomes for $24 \mathrm{~h}$. (C) M2 macrophages were treated with liposomes for 24 h. Each value represents the mean $\pm S D(n=3)$. ${ }^{*} p<0.05, * * p<0.01$, and $* * * p<0.001$ compared with the control group.

Abbreviations: Lipo, liposomes; PEG-Lipo, PEGylated liposomes; Man-PEG-Lipo, mannosylated liposomes; IL-4, Interleukin-4.

tumor growth and metastasis, immunosuppression, and angiogenesis. ${ }^{38,43}$ Therefore, a cancer immunotherapy that targets TAMs, including depleting or re-educating TAMs by immunomodulators, is a promising strategy.

Nanosized particulate carriers such as liposomes and nanoparticles, which are amenable to ligand-based active targeting, can accumulate in tumor tissue paving way for preferential tumoral localization of pharmaceutical molecules. ${ }^{39,44}$ This provides the capability to deliver immunomodulators to selectively eliminate or re-educate TAMs that infiltrate the tumor microenvironment. ${ }^{39}$ The knowledge gained concerning nanocarrier-mediated targeting of TAMs has highlighted the importance of understanding the interaction between nanocarriers and TAMs for the design and development of optimal drug delivery systems. ${ }^{39}$ The biodistribution of nanocarriers as well as their intrinsic immunostimulatory activity are profoundly affected by their surface characteristics. ${ }^{24}$ The mannose receptor is one of the most commonly targeted receptors, due to its overexpression of TAMs. ${ }^{45}$ Previous studies demonstrated that mannosylated liposomes have a distinct TAMs-targeting capacity as compared with conventional bare liposomes. ${ }^{17}$ To the best of our knowledge, the present study is the first report on the effect of mannosylated liposomes on macrophage activation and polarization.

As professional phagocytes, macrophages very efficiently take up extracellular materials including nanosized particles. ${ }^{46}$ To evaluate the macrophage biocompatibility of the liposomes, the in vitro cytotoxicity of the three liposomes was determined for RAW264.7 macrophages (Figure 4). The liposomes were not cytotoxic to macrophages and macrophage proliferation was stimulated at concentrations exceeding $500 \mu \mathrm{g} / \mathrm{mL}$, suggesting that all the liposomes have excellent biocompatibility. Additionally, the observation of enhanced macrophage proliferation in the presence of high concentrations of lipids suggests the possibility that the proliferation of macrophages within the immunosuppressive microenvironment of tumors could be greatly enhanced.

M2 macrophages express relatively high levels of mannose receptor (MR) on the cell surface, but there are few MRs on the surface of M1 macrophages. ${ }^{38}$ Based on these different expression profiles, the cellular uptake efficacy of liposomes was evaluated to determine whether endocytosis of liposomes was associated with MR. The results indicate that overexpression of MR significantly facilitated the cellular uptake of Man-PEG-Lipo in M2 macrophages and that the expression profile of MR modulated the cellular uptake of Man-PEG-Lipo before and after polarization (Figure 5). The comprehensive results of uptake, internalization mechanism, and intracellular trafficking support the conclusion that Man-PEG-Lipo may be internalized into M2 macrophages through an MR-mediated internalization pathway via caveolin-coated pits (Figure 7A) with subsequent localization in lysosomes (Figure 7B). This finding is consistent with the results of a previous study. ${ }^{25}$

The conventional two-dimensional monolayer model is deficient in providing the environmental cues that define a tumor microenvironment and is incapable of simulating the $3 \mathrm{D}$ localization of macrophages with respect to the tumor. ${ }^{30,47}$ 
A

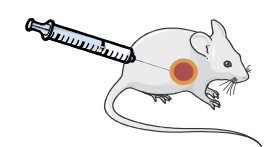

G422 cells were injected subcutaneously
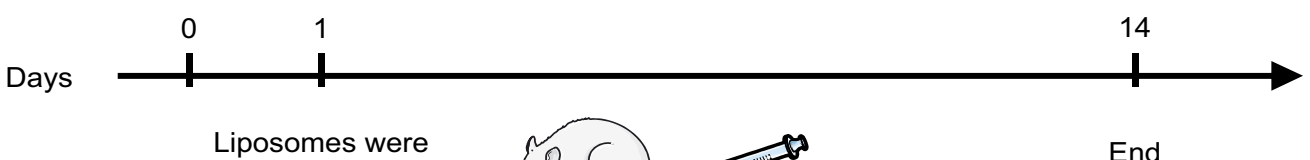
administrated per day
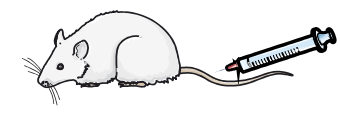

End

B

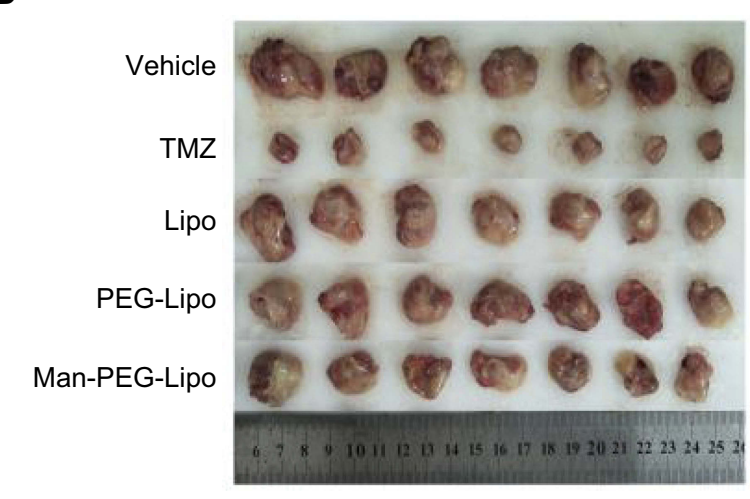

D

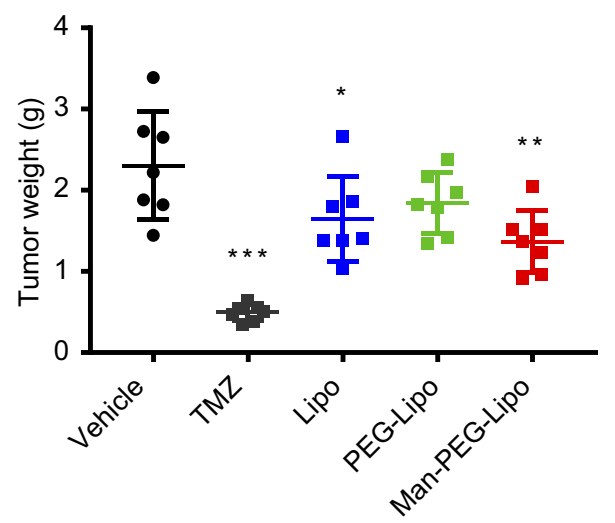

C

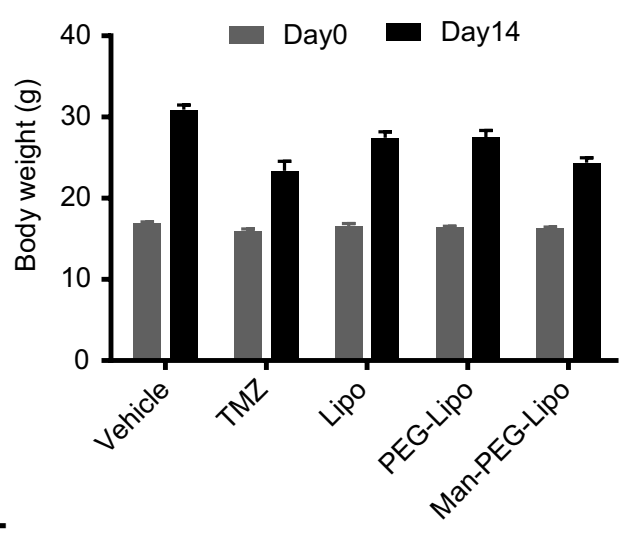

E

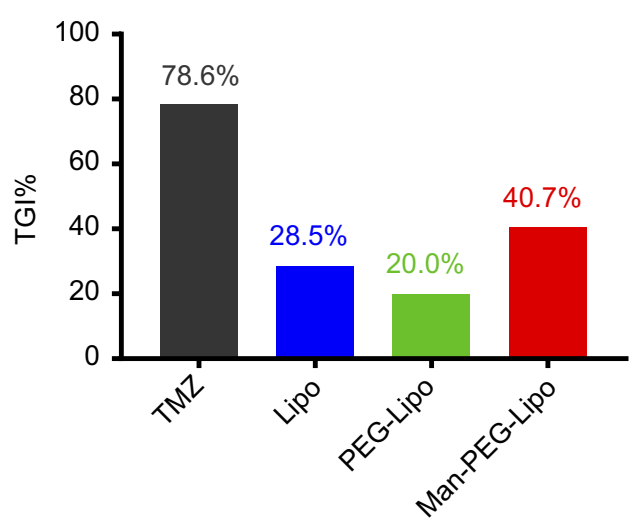

Figure 10 In vivo antitumor efficacy and safety evaluation of liposomes. (A) The schematic of treatment schedule. The images of excised tumors (B), body weight changes (C), tumor weight (D), and tumor growth inhibition ratio (TGI\%) (E) of murine G422 glioma tumor-bearing mice after the intravenous treatment of liposomes. Each value represents the mean $\pm S D(n=7)$. ${ }^{*} p<0.05$, ${ }^{*} p<0.01$, and ${ }^{*} * * p<0.001$ compared with the vehicle group.

Abbreviations: Lipo, liposomes; PEG-Lipo, PEGylated liposomes; Man-PEG-Lipo, mannosylated liposomes; TGI\%, tumor growth inhibition ratio.

Therefore, development of a 3D co-cultured spheroid model, which mimics the clinical observations of TAM localizations and subsequent functional performance, has profound value in evaluating the penetration and accumulation efficiency of nanocarriers within tumors. As reported in another co-culture model study, the incorporation of macrophages within breast cancer spheroids can convert macrophage differentiation toward an M2 phenotype. $^{30,48}$ In the present study, the results of $3 \mathrm{D}$ co-cultured spheroid penetration demonstrated the greater penetration efficiency of Man-PEG-Lipo compared to Lipo and PEG-Lipo (Figure 6C), indicating that the enhanced penetration efficiency of Man-PEG-Lipo could be associated with the elevated expression profile of MR in co-cultured 4T1 and RAW264.7 cells. Additionally, Man-PEG-Lipo exhibited enhanced tumor targeting efficiency in orthotopic 4T1 breast tumors as a result of TAMs-targeting effects (Figure 8).

M1 phenotype macrophages facilitate the secretion of pro-inflammatory cytokines and enhance the expression of 
A
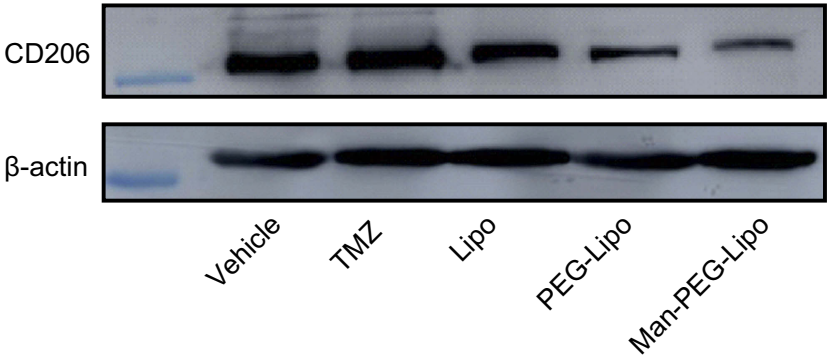

C

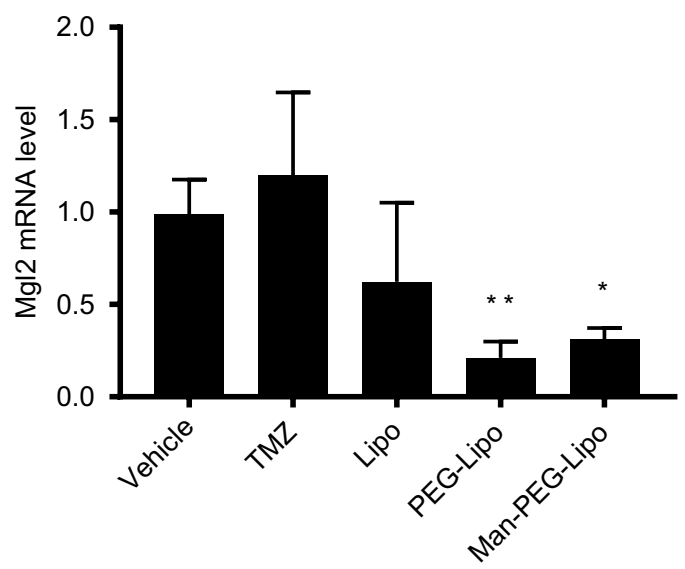

B

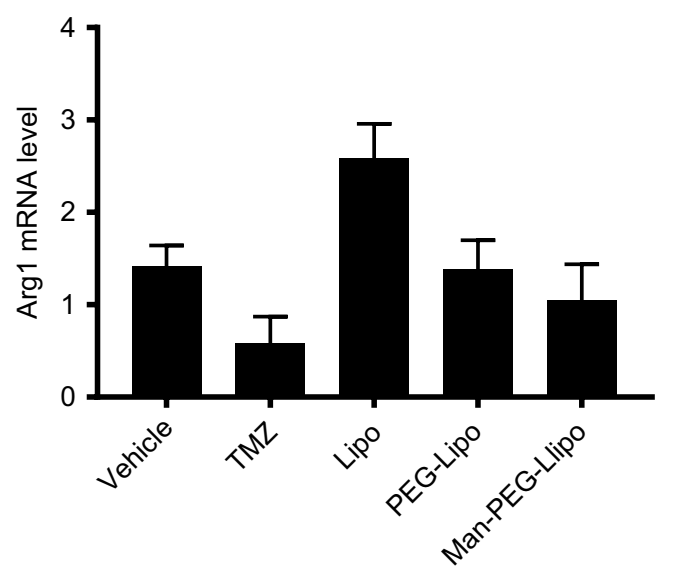

D

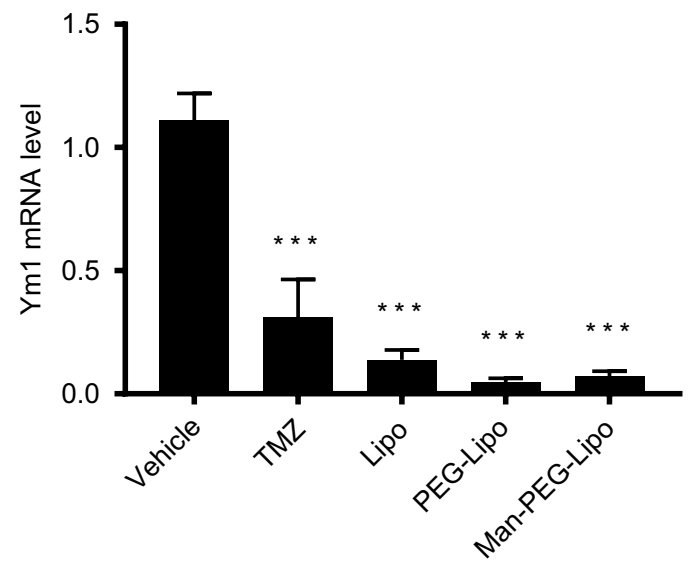

Figure II Man-PEG-Lipo promotes the polarization of TAMs. Murine G422 glioma tumor-bearing mice were treated with liposomes, and the tumors were analyzed by western blot for CD206 expression (A), and quantitative real-time PCR for Argl (B), Mgl2 (C), and Yml (D) mRNA expression. Each value represents the mean \pm SD $(n=3) . * p<0.05, * * p<0.01$, and $* * * p<0.001$ compared with the vehicle group.

Abbreviations: Lipo, liposomes; PEG-Lipo, PEGylated liposomes; Man-PEG-Lipo, mannosylated liposomes; TAMs, tumor-associated macrophages; CD206, cluster of differentiation 206; Argl, Arginase I; Mgl2, Macrophage galactose-type C-type lectin 2; Ym I, Chitinase 3-like 3.

co-stimulatory molecules, such as $\mathrm{CD} 86$, to promote the immunological response against tumors. On the contrary, M2 phenotype macrophages overexpress CD206 and abundantly produce anti-inflammatory cytokines to produce a protumoral effect. ${ }^{39}$ Therefore, the immunostimulatory activity of liposomes on macrophages was investigated by determining the pivotal features of polarized macrophages. Previous studies have shown that certain PEGylated liposomes (no drug loaded within), which are similar in size and composition to the Doxil® (PEGylated liposomal doxorubicin) drug carrier, could induce polarization of TAMs toward an M2-phenotype and then lead to tumor progress and immunosuppression in murine cancer models. ${ }^{40,41}$ However, the extent to which the findings of the above studies are broadly applicable to other nanoparticles or other types of tumors remains to be determined. There is substantial heterogeneity in physicochemical properties among different types of liposomes and in tumor biology among different types of tumors. $^{41}$ Since surface functionalization of nanocarriers with specific ligands has an important effect on the interactions between nanocarriers and biological targets, conventional bare liposomes, PEGylated liposomes, and mannosylated liposomes were developed and the immunostimulatory activity of these liposomes to macrophages evaluated in the present study. In contrast to previous studies mentioned above, ${ }^{40,41}$ Man-PEG-Lipo promoted the polarization of both M0 and M2 to M1 phenotype by enhancing the expression ratio of CD86/CD206 in the present study (Figure 


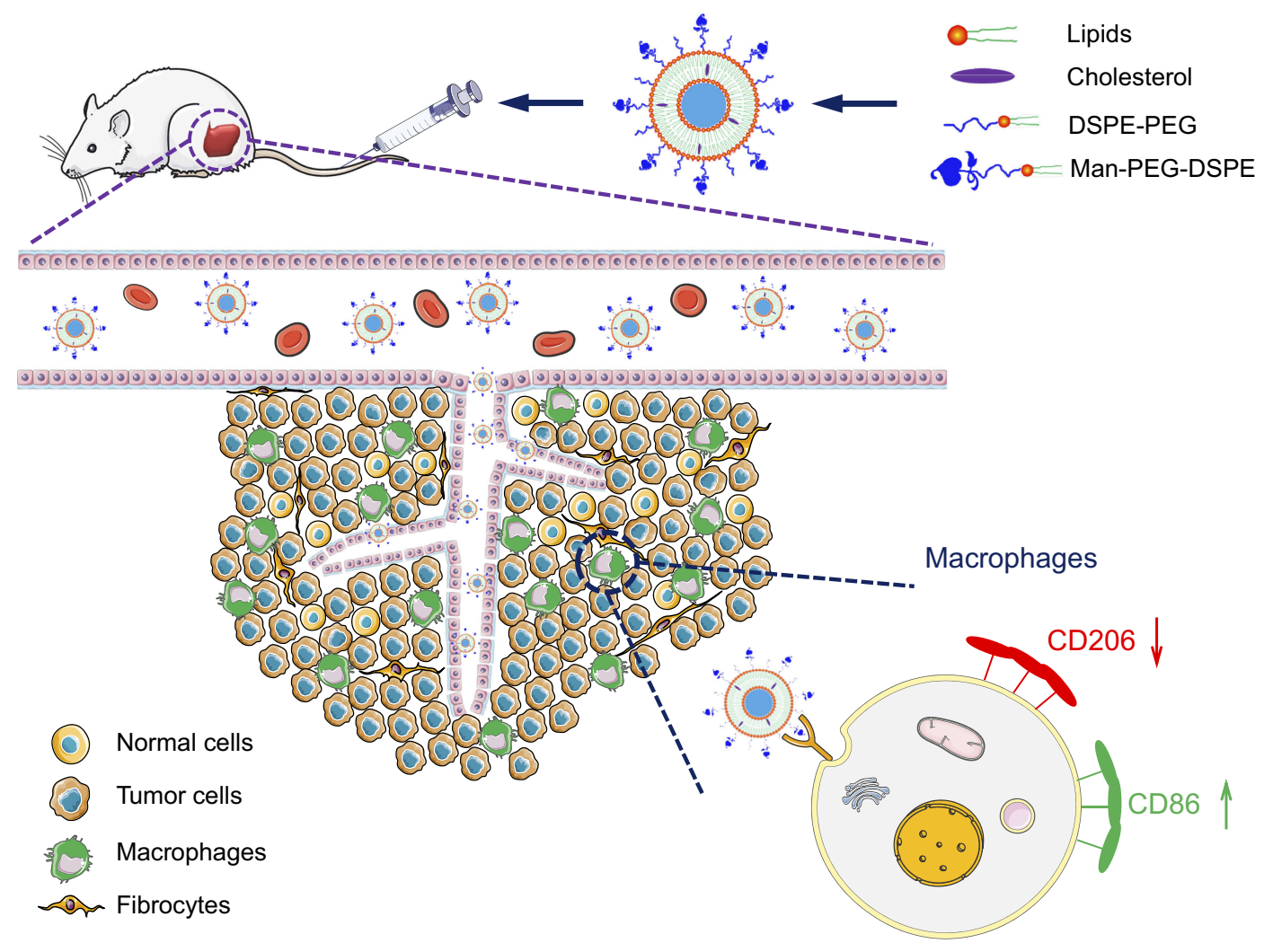

Scheme I The schematic diagram of Man-PEG-Lipo resetting TAMs.

Abbreviations: Man-PEG-Lipo, mannosylated liposomes; TAMs, tumor-associated macrophages.

9). Most significantly, Man-PEG-Lipo could inhibit G422 glioma tumor growth (Figure 10), which may be attributed to the polarization of TAMs, as evidenced by the reduction in the expression level of the TAMs surface receptor (CD206) and marker genes (Mgl2 and Ym1) (Figure 11). Previous studies reported that the nanoparticles modified with an established macromolecular ligand of MR could induce the clustering of MR on the surface of the macrophages, and then triggered intracellular signaling and consequently a phenotypic change. ${ }^{49}$ There was also evidence that glycocalyx-mimicking nanoparticles self-assembled by amphiphilic mannopyranoside polymer could promote the reversion of TAMs, which was mainly dependent on the downregulation of STAT6 phosphorylation and upregulation of NF- $\mathrm{kB}$ p65 phosphorylation. ${ }^{50}$ Based on the above findings, we hypothesized that liposomes modified with a ligand of MR named Man-PEG-Lipo may induce the clustering of MR on the surface of the M2 macrophages, and then suppress STAT6 and activate NF- $\mathrm{KB}$ phosphorylation, consequently promoting the polarization of M2 macrophages to the M1 phenotype. We will verify the specific mechanism in the later experiments. These results highlight the potential of Man-
PEG-Lipo in the design of a rational delivery system to enhance the antitumor immune efficacy of immunomodulators by inducing a shift from the M2 to the M1 phenotype.

\section{Conclusion}

A fundamental understanding of the interaction between nanocarriers and immune cells will facilitate the rational design and development of effective therapeutic strategies to enhance the efficacy of cancer immunotherapy. In the present study, cellular internalization and immunostimulatory activity of surface functionalization of liposomes, including conventional bare liposomes, PEGylated liposomes, and mannosylated liposomes, on TAMs were investigated. The data indicate that the potential of mannosylated liposomes in a delivery system that will enhance the antitumor immune efficacy of immunomodulators based on a shift from the M2 to the M1 phenotype.

\section{Acknowledgments}

This work was financially supported by the China Postdoctoral Science Foundation (No. 2017M620679), the National Natural Science Foundation of China (No. 
81402874), and the Natural Science Foundation of Beijing (No. 7162135).

\section{Disclosure}

The authors report no conflicts of interest in this work.

\section{References}

1. Junttila MR, de Sauvage FJ. Influence of tumour micro-environment heterogeneity on therapeutic response. Nature. 2013;501(7467): 346-354. doi:10.1038/nature 12626

2. Qian BZ, Pollard JW. Macrophage diversity enhances tumor progression and metastasis. Cell. 2010;141(1):39-51. doi:10.1016/j.cell.2010.03.014

3. Mantovani A, Schioppa T, Porta C, Allavena P, Sica A. Role of tumor-associated macrophages in tumor progression and invasion. Cancer Metastasis Rev. 2006;25(3):315-322. doi:10.1007/s10555006-9001-7

4. Komohara Y, Fujiwara Y, Ohnishi K, Takeya M. Tumor-associated macrophages: potential therapeutic targets for anti-cancer therapy. Adv Drug Deliv Rev. 2016;99(Pt B):180-185. doi:10.1016/j.addr.2015.11.009

5. Noy R, Pollard JW. Tumor-associated macrophages: from mechanisms to therapy. Immunity. 2014;41(1):49-61. doi:10.1016/j.immuni.2014.06.010

6. He H, Ghosh S, Yang H. Nanomedicines for dysfunctional macrophage-associated diseases. J Control Release. 2017;247:106-126. doi:10.1016/j.jconrel.2016.12.032

7. Zhang QW, Liu L, Gong CY, et al. Prognostic significance of tumor-associated macrophages in solid tumor: a meta-analysis of the literature. PLoS One. 2012;7(12):e50946. doi:10.1371/journal. pone.0050946

8. Bingle L, Brown NJ, Lewis CE. The role of tumour-associated macrophages in tumour progression: implications for new anticancer therapies. J Pathol. 2002;196(3):254-265. doi:10.1002/path.1027

9. Komohara Y, Jinushi M, Takeya M. Clinical significance of macrophage heterogeneity in human malignant tumors. Cancer Sci. 2014;105(1):1-8. doi:10.1111/cas.12314

10. Heusinkveld M, van der Burg SH. Identification and manipulation of tumor associated macrophages in human cancers. J Transl Med. 2011;9:216. doi:10.1186/1479-5876-9-216

11. Silva VL, Al-Jamal WT. Exploiting the cancer niche: tumor-associated macrophages and hypoxia as promising synergistic targets for nano-based therapy. J Control Release. 2017;253:82-96. doi:10.1016/j.jconrel. 2017.03.013

12. Liu Z, Xiong M, Gong J, et al. Legumain protease-activated TAT-liposome cargo for targeting tumours and their microenvironment. Nat Commun. 2014;5:4280.

13. Zhang X, Tian W, Cai X, et al. Hydrazinocurcumin encapsuled nanoparticles "re-educate" tumor-associated macrophages and exhibit anti-tumor effects on breast cancer following STAT3 suppression. PLoS One. 2013;8(6):e65896. doi:10.1371/journal.pone.0065896

14. Song X, Wan Z, Chen T, et al. Development of a multi-target peptide for potentiating chemotherapy by modulating tumor microenvironment. Biomaterials. 2016;108:44-56. doi:10.1016/j.biomaterials.2016.09.001

15. Piaggio F, Kondylis V, Pastorino F, et al. A novel liposomal Clodronate depletes tumor-associated macrophages in primary and metastatic melanoma: anti-angiogenic and anti-tumor effects. J Control Release. 2016;223:165-177. doi:10.1016/j.jconrel.2015.12.037

16. Yan Z, Wang F, Wen Z, et al. LyP-1-conjugated PEGylated liposomes: a carrier system for targeted therapy of lymphatic metastatic tumor. J Control Release. 2012;157(1):118-125. doi:10.1016/j. jconrel.2011.07.034

17. Tian B, Liu R, Chen S, et al. Mannose-coated gadolinium liposomes for improved magnetic resonance imaging in acute pancreatitis. Int J Nanomedicine. 2017;12:1127-1141. doi:10.2147/IJN.S123290
18. Mukherjee S, Baidoo JNE, Sampat S, et al. Liposomal TriCurin, a synergistic combination of curcumin, epicatechin gallate and resveratrol, repolarizes tumor-associated microglia/ macrophages, and eliminates glioblastoma (GBM) and GBM stem cells. Molecules. 2018;23(1). doi:10.3390/molecules23010201

19. Zhou S, Zhang T, Peng B, et al. Targeted delivery of epirubicin to tumor-associated macrophages by sialic acid-cholesterol conjugate modified liposomes with improved antitumor activity. Int $J$ Pharm. 2017;523(1):203-216. doi:10.1016/j.jpharm.2017.03.034

20. Chellat F, Merhi Y, Moreau A, Yahia L. Therapeutic potential of nanoparticulate systems for macrophage targeting. Biomaterials. 2005;26(35):7260-7275. doi:10.1016/j.biomaterials.2005.05.044

21. Wang D, Phan N, Isely C, Bruene L, Bratlie KM. Effect of surface modification and macrophage phenotype on particle internalization. Biomacromolecules. 2014;15(11):4102-4110. doi:10.1021/ bm5011382

22. Zanganeh S, Hutter G, Spitler R, et al. Iron oxide nanoparticles inhibit tumour growth by inducing pro-inflammatory macrophage polarization in tumour tissues. Nat Nanotechnol. 2016;11 (11):986-994. doi:10.1038/nnano.2016.168

23. Pal R, Chakraborty B, Nath A, et al. Noble metal nanoparticle-induced oxidative stress modulates tumor associated macrophages (TAMs) from an M2 to M1 phenotype: an in vitro approach. Int Immunopharmacol. 2016;38:332-341. doi:10.1016/j. intimp.2016.06.006

24. Fuchs AK, Syrovets T, Haas KA, et al. Carboxyl- and amino-functionalized polystyrene nanoparticles differentially affect the polarization profile of M1 and M2 macrophage subsets. Biomaterials. 2016;85:78-87. doi:10.1016/j.biomaterials.2016.01.064

25. Su L, Zhang W, Wu X, et al. Glycocalyx-mimicking nanoparticles for stimulation and polarization of macrophages via specific interactions. Small. 2015;11(33):4191-4200. doi:10.1002/smll.201403838

26. Bygd HC, Forsmark KD, Bratlie KM. Altering in vivo macrophage responses with modified polymer properties. Biomaterials. 2015;56:187-197. doi:10.1016/j.biomaterials.2015.03.042

27. Lunov O, Syrovets T, Loos C, et al. Differential uptake of functionalized polystyrene nanoparticles by human macrophages and a monocytic cell line. ACS Nano. 2011;5(3):1657-1669. doi:10.1021/nn2000756

28. Zhang C, Yu X, Gao L, et al. Noninvasive imaging of CD206-positive M2 macrophages as an early biomarker for post-chemotherapy tumor relapse and lymph node metastasis. Theranostics. 2017;7(17):4276-4288. doi:10.7150/thno.20999

29. Xu Z, Ramishetti S, Tseng YC, Guo S, Wang Y, Huang L. Multifunctional nanoparticles co-delivering Trp2 peptide and $\mathrm{CpG}$ adjuvant induce potent cytotoxic T-lymphocyte response against melanoma and its lung metastasis. J Control Release. 2013;172 (1):259-265. doi:10.1016/j.jconrel.2013.08.021

30. Tevis KM, Cecchi RJ, Colson YL, Grinstaff MW. Mimicking the tumor microenvironment to regulate macrophage phenotype and assessing chemotherapeutic efficacy in embedded cancer cell/macrophage spheroid models. Acta Biomater. 2017;50:271-279. doi:10.1016/j.actbio.2016.12.037

31. Ruan GX, Chen YZ, Yao XL, et al. Macrophage mannose receptor-specific gene delivery vehicle for macrophage engineering. Acta Biomater. 2014;10(5):1847-1855. doi:10.1016/j.actbio.2014.01.012

32. Keswani RK, Lazebnik M, Pack DW. Intracellular trafficking of hybrid gene delivery vectors. J Control Release. 2015;207:120-130. doi:10.1016/j.jconrel.2015.04.015

33. Hill AA, Reid Bolus W, Hasty AH. A decade of progress in adipose tissue macrophage biology. Immunol Rev. 2014;262(1):134-152. doi:10.1111/ imr.12216

34. Bilzer M, Roggel F, Gerbes AL. Role of Kupffer cells in host defense and liver disease. Liver Int. 2006;26(10):1175-1186. doi:10.1111/ j.1478-3231.2006.01342.x 
35. Stahl PD. The macrophage mannose receptor: current status. Am J Respir Cell Mol Biol. 1990;2(4):317-318. doi:10.1165/ajrcmb/ 2.4.317

36. Lai C, Li C, Luo X, et al. Use of dual-ligand modification in kupffer cell-targeted liposomes to examine the contribution of kupffer cells to accelerated blood clearance phenomenon. Mol Pharm. 2018;15 (7):2548-2558. doi:10.1021/acs.molpharmaceut.8b00042

37. Zheng $\mathrm{H}$, Li J, Wang $\mathrm{M}$, et al. Exhausting tumor associated macrophages with sialic acid-polyethyleneimine-cholesterol modified liposomal doxorubicin for enhancing sarcoma chemotherapy. Int J Pharm. 2019;558:187-200. doi:10.1016/j. ijpharm.2019.01.005

38. Mantovani A, Sozzani S, Locati M, Allavena P, Sica A. Macrophage polarization: tumor-associated macrophages as a paradigm for polarized M2 mononuclear phagocytes. Trends Immunol. 2002;23 (11):549-555.

39. Singh Y, Pawar VK, Meher JG, et al. Targeting tumor associated macrophages (TAMs) via nanocarriers. $J$ Control Release. 2017;254:92-106. doi:10.1016/j.jconrel.2017.03.395

40. Sabnani MK, Rajan R, Rowland B, et al. Liposome promotion of tumor growth is associated with angiogenesis and inhibition of antitumor immune responses. Nanomedicine. 2015;11(2):259-262. doi:10.1016/j. nano.2014.08.010

41. Rajan R, Sabnani MK, Mavinkurve V, et al. Liposome-induced immunosuppression and tumor growth is mediated by macrophages and mitigated by liposome-encapsulated alendronate. $J$ Control Release. 2018;271:139-148. doi:10.1016/j.jconrel.2017.12.023

42. Smyth MJ, Ngiow SF, Ribas A, Teng MWL. Combination cancer immunotherapies tailored to the tumour microenvironment. Nat Rev Clin Oncol. 2015;13:143. doi:10.1038/nrclinonc.2015.209
43. Chen D, Xie J, Fiskesund R, et al. Chloroquine modulates antitumor immune response by resetting tumor-associated macrophages toward M1 phenotype. Nat Commun. 2018;9(1):873. doi:10.1038/s41467-01803225-9

44. Bobo D, Robinson KJ, Islam J, Thurecht KJ, Corrie SR. Nanoparticlebased medicines: a review of FDA-approved materials and clinical trials to date. Pharm Res. 2016;33(10):2373-2387. doi:10.1007/s11095-0161958-5

45. Ngambenjawong C, Gustafson HH, Pun SH. Progress in tumor-associated macrophage (TAM)-targeted therapeutics. $A d v$ Drug Deliv Rev. 2017;114:206-221. doi:10.1016/j.addr.2017.04.010

46. Lunov O, Syrovets T, Röcker C, et al. Lysosomal degradation of the carboxydextran shell of coated superparamagnetic iron oxide nanoparticles and the fate of professional phagocytes. Biomaterials. 2010;31(34):9015-9022. doi:10.1016/j.biomaterials.2010.08.003

47. Kuen J, Darowski D, Kluge T, Majety M. Pancreatic cancer cell/ fibroblast co-culture induces M2 like macrophages that influence therapeutic response in a 3D model. PLoS One. 2017;12(7): e0182039. doi:10.1371/journal.pone.0182039

48. Leonard F, Curtis LT, Ware MJ, et al. Macrophage polarization contributes to the anti-tumoral efficacy of mesoporous nanovectors loaded with albumin-bound paclitaxel. Front Immunol. 2017;8:693. doi:10.3389/fimmu.2017.00693

49. Gan J, Dou Y, Li Y, et al. Producing anti-inflammatory macrophages by nanoparticle-triggered clustering of mannose receptors. Biomaterials. 2018;178:95-108. doi:10.1016/j.biomaterials.2018.06.015

50. Zhang Y, Wu L, Li Z, et al. Glycocalyx-mimicking nanoparticles improve anti-PD-L1 cancer immunotherapy through reversion of tumor-associated macrophages. Biomacromolecules. 2018;19 (6):2098-2108. doi:10.1021/acs.biomac.8b00305 


\section{Supplementary material}

A

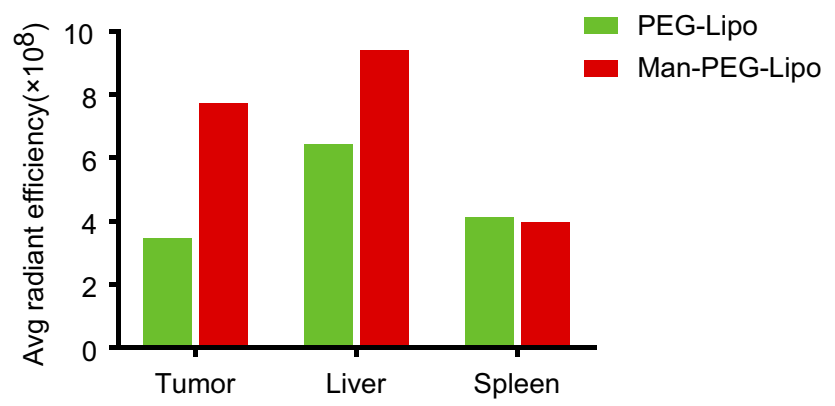

B

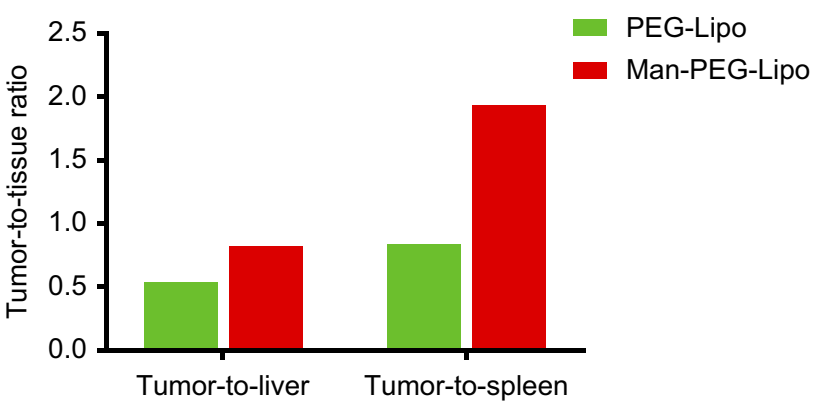

Figure SI Ex vivo average radiant efficiency of excised tissues and tumors at $24 \mathrm{~h}$ post-injection. The average radiant efficiency (A) and tumor-to-tissue ratios (B) measured from the fluorescence images of excised tissues and tumors.

Abbreviations: PEG-Lipo, PEGylated liposomes; Man-PEG-Lipo, mannosylated liposomes.

\section{Publish your work in this journal}

The International Journal of Nanomedicine is an international, peerreviewed journal focusing on the application of nanotechnology in diagnostics, therapeutics, and drug delivery systems throughout the biomedical field. This journal is indexed on PubMed Central, MedLine, CAS, SciSearch ${ }^{\circledR}$, Current Contents ${ }^{\circledR} /$ Clinical Medicine,
Journal Citation Reports/Science Edition, EMBase, Scopus and the Elsevier Bibliographic databases. The manuscript management system is completely online and includes a very quick and fair peer-review system, which is all easy to use. Visit http://www.dovepress.com/ testimonials.php to read real quotes from published authors. 